Sección Agrícola / Agriculture

Artículos de investigación / Research paper

\title{
Repelencia de adultos de mosca blanca Trialeurodes vaporariorum (Heteroptera: Aleyrodidae) con clavo y pimienta
}

\author{
Repellency of adults of whitefly Trialeurodes vaporariorum (Heteroptera: Aleyrodidae) \\ with clove and pipper
}

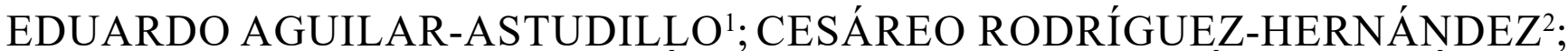 HIRAM BRAVO-MOJICA ${ }^{3}$; RAMÓN MARCOS SOTO-HERNÁNDEZ ${ }^{4}$; NÉSTOR BAUTISTA-MARTIINEZ ${ }^{5}$; FRANCISCO GUEVARA-HERNÁNDEZ ${ }^{6}$}

\begin{abstract}
${ }^{1}$ Doctor en Ciencias, Facultad de Ciencias Agronómicas, Universidad Autonoma de Chiapas, Ocozocoautla, Chiapas, México, guerr2012@hotmail.es, https:// orcid.org/0000-0001-8057-8782. ${ }^{2}$ Doctor en Ciencias, Posgrado de Fitosanidad, Colegio de Postgraduados, Campus Montecillo, Texcoco, Estado de México, México,crhernan@colpos.mx, https://orcid.org/0000-0001-7137-3697. ${ }^{3}$ Doctor en Ciencias, Posgrado de Fitosanidad, Colegio de Postgraduados, Campus Montecillo, Texcoco, Estado de México, México, bravomj@colpos.mx, https://orcid.org/0000-0002-6135-9012. ${ }^{4}$ Doctor en Ciencias, Posgrado de Botanica, Colegio de Postgraduados, Campus Montecillo, Texcoco, Estado de México, México, msoto@colpos.mx https://orcid.org/0000-0001-8577-7991.5 Doctor en Ciencias, Posgrado de Fitosanidad, Colegio de Postgraduados, Campus Montecillo, Texcoco, Estado de México, México, nestor@colpos.mx, https://orcid. org/0000-0002-1332-2565. ${ }^{6}$ Doctor en Ciencias, Facultad de Ciencias Agronómicas, Universidad Autonoma de Chiapas, Ocozocoautla, Chiapas, México, fragueher@prodigy.net.mx, https://orcid.org/0000-0002-1444-6324.
\end{abstract}

\begin{abstract}
Autor para correspondencia
Cesáreo Rodríguez-Hernández. Doctor en Ciencias. Posgrado de Fitosanidad. Colegio de Postgraduados, Campus Montecillo, Carretera Federal México-Texcoco km 81,5 C. P. 56230, Texcoco, Estado de México, México, crhernan@colpos.mx, https://orcid.org/0000-00017137-3697.

Citación sugerida

AGUILAR-ASTUDILLO, E.; RODRÍGUEZHERNÁNDEZ, C.; BRAVO-MOJICA, H.; SOTO-HERNÁNDEZ, R. M.; BAUTISTAMARTÍNEZ, N.; GUEVARA-HERNÁNDEZ, F. 2020. Repelencia de adultos de mosca blanca Trialeurodes vaporariorum (Heteroptera: Aleyrodidae) con clavo y pimienta. Revista Colombiana de Entomología 46 (2): e7520. https://doi.org/10.25100/socolen.v46i2.7520
\end{abstract}

Recibido: 14-feb-2019

Aceptado: 13-ene-2020

Publicado: 28-dic-2020

Revista Colombiana de Entomología ISSN (Print): 0120-0488

ISSN (On Line): 2665-4385

https://revistacolombianaentomologia.univalle.edu.co

Open access

(c) (i) (2) (2) BY-NC-SA 4.0

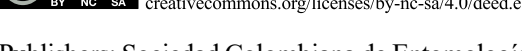

Publishers: Sociedad Colombiana de Entomología SOCOLEN (Bogotá, D. C., Colombia) https://www.socolen.org.co

Universidad del Valle (Cali, Colombia)

https://www.univalle.edu.co

(C) 2020 Sociedad Colombiana de Entomología SOCOLEN y Universidad del Valle
Resumen: En la mosca blanca Trialeurodes vaporariorum, plaga de importancia económica en hortalizas, su manejo con insecticidas sintéticos ha generado resistencia con efectos adversos para el ambiente. En la búsqueda de alternativas de manejo, se planteó esta investigación con el objetivo de cuantificar la repelencia de adultos con extractos crudos (metanol, etanol, diclorometano y hexano), aceites esenciales (AE) y compuestos mayoritarios de clavo y pimienta. En un vaso con $10 \mathrm{~mL}$ de agua, se colocó una hoja de tomate tratada a concentraciones de 1,0 a $0,000001 \%$ y se introdujeron 20 adultos de $2 \mathrm{~d}$ de edad. Luego, de las 3 a las $72 \mathrm{~h}$, se cuantificaron los adultos posados y por diferencia se determinó la repelencia. Los extractos crudos, AE y compuestos mayoritarios de clavo y pimienta repelieron entre 32,5 y 90,2 \% la población de adultos de las 3 a las 72 h. Los AE de clavo y pimienta presentan mejor efecto repelente que los extractos crudos y compuestos secundarios, ya que lograron repeler de 46,2 a $100 \%$; el AE de clavo a la concentración de $1,0 \%$ logró $100 \%$ de repelencia a las 48 y $72 \mathrm{~h}$ y el AE de pimienta alcanza $97,5 \%$ a las $72 \mathrm{~h}$. En la práctica, se debe utilizar extracto crudo etanólico de ambas plantas a la concentración de 0,000001 $\%$ ya que logran repeler entre 38,7 y $87,5 \%$ de adultos, de las 3 a las $72 \mathrm{~h}$, y su efecto repelente persiste; además, es más fácil de conseguir, más seguro para el operador, distribuidor y consumidor; y está considerado en las normas de agricultura orgánica como solvente para la extracción de compuestos secundarios.

Palabras clave: Extractos, aceites, compuestos, acuosos, crudos, condimento, plantas aromáticas.

Abstract: The whitefly Trialeurodes vaporariorum, an economically important pest in vegetables, is managed with synthetic pesticides and has generated resistance with adverse effects on the environment. In the search for alternative management, adult repellence with raw extracts (methanol, ethanol, dichloromethano, and hexane) essential oils, and major compounds of clove and pepper was tested. For this, a treated tomato leaf was placed at concentrations of 1.0 to $0.000001 \%$ within a $1 \mathrm{~L}$ beaker containing a $10 \mathrm{~mL}$ flask with water. Subsecuently, 20 adults of $2 \mathrm{~d}$ of age were introduced. After 3 to $72 \mathrm{~h}$, the posed adults were quantified, and repellence was determined from the difference. The raw extracts, essential oils, and major compounds of clove and pepper repel from 32.5 to $90.2 \%$ of the adult population in 3 to $72 \mathrm{~h}$. The cloves and pepper EO have a better repellent effect than raw extracts and secondary compounds, since they manage to repel $46.2 \%$ to $100 \%$. The clove EO at the concentration of $1.0 \%$ achieves $100 \%$ repellency at 48 and $72 \mathrm{~h}$ after application, while the pepper EO reaches $97.5 \%$ at $72 \mathrm{~h}$ after application; in practice, ethanolic crude extract of both plants should be used at a concentration of $0.000001 \%$ since they can repel 38.7 to $87.5 \%$ of adults, from 3 to $72 \mathrm{~h}$, and its repellent effect is maintained; also, it is easier to acquire, safer for the operator, distributor and consumer; and is considered a solvent for the extraction of secondary compounds in organic farming standards.

Keywords: Extracts, oils, compound, aqueous, raw, seasoning, aromatic plants. 


\section{Introducción}

La mosca blanca Trialeurodes vaporariorum Westwood, 1856 (Hemiptera: Aleyrodidae) presenta tres estados biológicos, (huevo, ninfa y adulto), pero en ninfa pasa por cuatro instares, siendo el último una etapa en que no se alimenta y se conoce como "pupa". Completa su ciclo de vida en 14 a $28 \mathrm{~d}$, de acuerdo a las condiciones de alimento y temperatura, presenta de 11 a 15 generaciones por ciclo de cultivo y por su alta capacidad reproductiva alcanza altas densidades poblacionales; la mosca blanca se distribuye ampliamente en las regiones tropicales, subtropicales y zonas templadas del mundo. Además, se considera transmisor de virus: las ninfas y adultos en su proceso de alimentación causan daños directos en los cultivos tanto a cielo abierto como en invernadero, ya que extraen nutrimentos e inducen reacciones bioquímicas en las plantas (Gerling 2002; Cardona et al. 2005; Ortíz et al. 2010); cuando están expuestas a temperaturas mayores a $30{ }^{\circ} \mathrm{C}$ sintetizan proteínas de choque térmico y acumulan sorbitol para regular la temperatura corporal (Wolfe et al. 1998; Salvucci 2000). El uso inadecuado de insecticidas sintéticos ha provocando la generación de resistencia, con efectos adversos al ambiente y la salud humana (Xiao-Wei et al. 2017). Los estados ninfales y el adulto son más susceptibles que los huevos y las "pupas" (Gerling 2002); sin embargo, el adulto es capaz de pasar sus partes bucales a través de una gota de insecticida hasta el tejido vegetal sin ingerir insecticida.

Por otro lado, las plantas son laboratorios químicos naturales, ya que producen sustancias bioactivas que interfieren en la alimentación, oviposición y refugio de varias especies de insectos fitófagos. Se considera que su aplicación en forma de extractos y aceites esenciales los hace más efectivos que los compuestos secundarios puros, esto se debe a las sustancias que conforman el extracto. Algunas actúan como potenciadoras $\mathrm{o}$, simplemente, como sinergistas, causan repelencia, disuasión de alimentación y oviposición, e inhiben la eclosión de huevos (Rodríguez 2004; Won-Sik et al. 2006; Guan et al. 2007; Pitasawat et al. 2007; Islam et al. 2009). En particular, los extractos orgánicos y aceites esenciales de clavo Syzygium aromaticum (L.) Merr. y L. M. Perry, 1939 (Myrtales: Myrtaceae) y pimienta Pimenta dioica (L.) Merr., 1947 (Myrtales: Myrtaceae), se han reportado como insecticidas de contacto y fumigante. Los extractos acuosos, crudos y el aceite esencial de clavo $S$. aromaticum están reportados como insecticidas e insectistáticos contra la cucaracha Periplaneta americana L., 1758 (Blattodea: Blattidae) (Sharawi et al. 2013), gorgojos del maíz Rhyzopertha dominica Fabricius, 1792 (Coleoptera: Bostrichidae) y Sitophilus oryzae L., 1763 (Coleoptera: Curculionidae) (Sighamony et al. 1986), Tribolium castaneum Herbst, 1797 (Coleoptera: Tenebrionidae) y Sitophilus zeamais Motsch., 1855 (Coleoptera: Curculionidae) (Ho et al. 1994), Bruchidius incarnatus Boheman, 1833 (Coleoptera: Bruchidae) (Fouad 2013). Los extractos acuosos y orgánicos de botones florales de clavo al $0,4 \%$ con 24 h de exposición inhiben $80 \%$ la oviposición de la mosca blanca Bemisia tabaci Genn., 1889 (Hemiptera: Aleyrodidae) (Cruz-Estrada et al. 2015).

La acción de los aceites esenciales en los insectos se debe a la inhibición de la acetilcolinesterasa; son neurotóxicos y afectan los receptores de la octopamina del sistema nervioso octopaminérgico, causan inhibición de la alimentación y repelencia (Grundy y Still 1985; Miyazawa et al. 1997; Enan 2005). El aceite esencial de clavo, afecta los parámetros biológicos de los insectos (González-Coloma 2006; Isikber et al. 2006; Nathan et al. 2008), actúa como fumigante, contacto, repelente e inhibidor de la alimentación y reproducción (WonSik et al. 2006; Guan et al. 2007; Pitasawat et al. 2007; Islam et al. 2009). Con aplicaciones de aceite esencial de botones florales de clavo contra la mosca blanca $B$. tabaci y por acción fumigante se obtiene la $\mathrm{CL}_{50}$ de $0,001354 \%$ en 24 h de exposición (Chao et al. 2014); con las concentraciones de $0,00023 \%$ y $0,00093 \%$ de aceite esencial de botones florales y hojas, ocasionan 90 y $58 \%$ de mortalidad de adultos de mosca blanca T. vaporariorum con $24 \mathrm{~h}$ de exposición (Won-Il et al. 2003).

En el manejo de la mosca blanca $T$. vaporariorum de 2 a 4 d de edad con aceite esencial comercial de pimienta $P$. dioca a concentraciones de 0,000093 y $0,00023 \%$, con $24 \mathrm{~h}$ de exposición, causaron mortalidades de 18,0 y $100 \%$; con Pimenta racemosa (Mill.) J. W. Moore, 1933 (Mytales: Myrtaceae), las mortalidades fueron de 20,0 y $100 \%$, respectivamente (Won-Il et al. 2003). No obstante, son pocos los trabajos registrados sobre el uso de los compuestos de estas plantas y amerita investigación que considere la extracción con varios disolventes, se evalúen los aceites esenciales y los compuestos secundarios mayoritarios a diferentes concentraciones. En el presente estudio se cuantificó la repelencia de adultos de mosca blanca $T$. vaporariorum, con siete concentraciones de extractos crudos (metanol, etanol, diclorometanol y hexano), aceites esenciales y compuestos mayoritarios de clavo y pimienta de las 3 a las $72 \mathrm{~h}$ aplicados en una hoja de tomate.

\section{Materiales y métodos}

Esta investigación se realizó en el área de insecticidas vegetales del programa de Fitosanidad Entomología-Acarología y en el laboratorio de Fitoquímica del programa de Botánica del Colegio de Postgraduados, Campus Montecillo, Montecillo, Texcoco, Estado de México; y en el laboratorio de Sanidad Vegetal de la Facultad de Ciencias Agronómicas Campus V, de la Universidad Autónoma de Chiapas, Villaflores, Chiapas, México.

Obtención de extractos, aceites y compuestos secundarios. Los frutos de clavo y pimienta, adquiridos en tiendas comerciales de especias de la central de abasto en Tuxtla Gutiérrez, Chiapas, se molieron con un molino eléctrico hasta obtener un polvo fino. Se colocaron $400 \mathrm{~g}$ en cada frasco de $5 \mathrm{~L}$ con metanol, etanol $98 \%$, diclorometano y hexano, se aforaron y se dejó en maceración a temperatura ambiente por $48 \mathrm{~h}$. Después de colar, se evaporó el disolvente utilizando un roto-evaporador marca Buchi modelo R-114, por tres veces durante $4 \mathrm{~h}, \mathrm{y}$ el residuo se dejó secar a temperatura ambiente. Se obtuvieron así los extractos crudos en metanol, etanol, diclorometano y hexano de clavo y de pimienta, los cuales se colocaron en frascos color ámbar de $50 \mathrm{~mL}$ a temperaturta de $4{ }^{\circ} \mathrm{C}$ hasta su utilización.

Con la extracción del aceite esencial de clavo y pimienta, se colocaron $400 \mathrm{~g}$ de polvo en un matraz de bola con capacidad de $5 \mathrm{~L}$ con $1 \mathrm{~L}$ de agua destilada. Se llevó a ebullición y se obtuvo la hidrodestilación. Por un periodo de $3 \mathrm{~h}$, el vapor de agua arrastró los compuestos de clavo y pimienta, que al pasar por el sistema de enfriamiento se condensaron y se recolectó junto con la fracción acuosa. El aceite esencial y el hidrolato (fase acuosa) se separaron, utilizando un embudo de separación. El aceite esencial de clavo y pimienta se almacenaron en frascos de vidrio de color ámbar para su posterior utilización. Los tres compuestos mayoritarios de los extractos crudos y aceite esencial de clavo y pimienta, eugenol, metil 
eugenol y $\beta$-cariofileno se adquirieron en tiendas comerciales (Sigma-Aldrich, México); a las concentraciones de 99, 98 y $80 \%$, respectivamente.

Cría de mosca blanca. Se recolectaron adultos de mosca blanca en cultivos de tomate en las localidades de Villa Hidalgo y 16 de septiembre, Villaflores, Chiapas. La especie se determinó con las claves de Carapia y Castillo-Gutiérrez (2013), y fue corroborada por la Dra. Laura Delia Ortega Arenas, investigadora titular de insectos vectores del Colegio de Postgraduados, Campus Montecillo. Los adultos de mosca blanca se confinaron en camáras entomológicas de $2 \mathrm{~m}^{3}$, cubiertas con tela de organza, donde se colocaron plantas de tomate var. Río Grande de 20 - 30 d de edad para alimentación y oviposición. La incubación de huevos, el desarrollo de ninfas y la emergencia de nuevos adultos se realizaron en otras camáras y cuando se requirieron ejemplares para los bioensayos de repelencia de adultos, se sacaron las plantas que tenían ninfas de cuarto instar "pupas" y se colocaron en camára separada para la emergencia de adultos durante $2 \mathrm{~d}$ hasta obtener adultos de $2 \mathrm{~d}$ de edad.

Bioensayos de repelencia. La evaluación de repelencia de adultos de mosca blanca $T$. vaporariorum se realizó con el método propuesto por Ortega y Schuster (2000), con modificaciones en cuanto al tamaño del contenedor y del sustrato. En estos bioensayos se utilizaron vasos de plástico transparente con capacidad de $1 \mathrm{~L}$, colocando en la parte de la tapa tela de organza para permitir la ventilación, y en el interior del vaso un frasco tipo gotero de $10 \mathrm{~mL}$ con agua, para mantener la turgencia de las hojas de tomate. Las hojas tratadas por inmersión en el extracto crudo, aceite esencial, o compuesto secundario de clavo y pimienta a las concentraciones de 1,0 ; 0,$1 ; 0,01 ; 0,001 ; 0,0001 ; 0,00001$ y $0,000001 \%$, más los testigos con emulsificante (Tween 80) y absoluto (agua). Luego, se dejó secar para eliminar el exceso de humedad. En cada bioensayo se colocaron cuatro repeticiones por tratamiento; cada vaso se consideró como la unidad experimental donde se introdujeron 20 adultos de mosca blanca de $2 \mathrm{~d}$ de edad, sin sexar, con $1 \mathrm{~h}$ de ayuno; para esto se utilizaron aspiradores manuales elaborados con pipetas Pasteur de punta corta. A los 30 min de establecer el experimento se registró el número de adultos posados, siguiendo las observaciones cada hora por 6 $\mathrm{h}$ consecutivas y luego a las $12,24,48$ y $72 \mathrm{~h}$. Para determinar el porcentaje de moscas no posadas se calculó por diferencia de los posados y en consideración a la población total. Los datos que se reportan en este trabajo son a partir de las 3 y $3,5 \mathrm{~h}$ respetando el tiempo de ambientación de la mosca blanca.

Análisis estadístico. A los porcentajes de repelencia de adultos, se aplicó la prueba de los supuestos de normalidad Shapiro-Wilks y de homogeneidad con la prueba de Levene, por tratamiento y tiempo de muestreo. Cuando los datos cumplieron los supuestos se analizó con estadística paramétrica y para la comparación de medias se utilizó la prueba de Tukey al 0,5 $\%$. Pero cuando no cumplieron los supuestos de normalidad, los datos se analizaron con estadística no paramétrica utilizando la prueba de Kruskal Wallis y las medias se compararon con los promedios de rangos con valor de $\mathrm{P}=0,05$ (Di Rienzo et al. 2013).

\section{Resultados y discusión}

Repelencia de adultos de mosca blanca. En los bioensayos preliminares se obtuvo fitotoxicidad de las hojas de tomate con la concentración de $10,0 \%$ de los extractos crudos, aceite esencial y compuestos puros de clavo y pimienta.

Extractos crudos de clavo. La repelencia provocada por el extracto crudo en metanol de clavo en adultos de mosca blanca va de 32,5 a $96,2 \%$ de las 3 a las $72 \mathrm{~h}$ con las concentraciones de 1,0 a $0,000001 \%$, sin correlación con la concentración. $\mathrm{Al}$ aumentar el tiempo después de la aplicación, la efectividad no decrece, se mantiene; en los tiempos de 3 y $72 \mathrm{~h}$ se obtienen 84,1 y $79,7 \%$ de repelencia promedio sin diferencia estadística entre las concentraciones y por tiempos de muestreo. La concentración más efectiva se considera $0,000001 \%$ por ocasionar de 55,0 a 96,2\% de repelencia (Tabla 1 ).

En el extracto crudo en etanol de clavo el mayor efecto repelente de la mosca blanca se obtuvo con la concentración del $1,0 \%$ en todos los tiempos de muestreo, que va de 68,7 a $100 \%$, y estadísticamente es diferente a las demás concentraciones excepto a las $72 \mathrm{~h}$ (Tabla 2). Desde el inicio de la toma de datos $(3,5 \mathrm{~h})$, el extracto en etanol de clavo presentó en promedio $74,9 \%$ de efectividad, y a las $72 \mathrm{~h}$ alcanzó

Tabla 1. Repelencia (\%) de adultos de mosca blanca Trialeurodes vaporariorum mediante extracto metanólico de clavo Syzygium aromaticum a concentraciones de 1,0 a $0,000001 \%$.

\begin{tabular}{|c|c|c|c|c|c|c|c|c|c|c|c|c|}
\hline Tiempo & 3 & & 4 & 5 & & & & 1 & & 24 & 48 & $72 \mathrm{~h}$ \\
\hline Conc. (\%) & ${ }^{*} \boldsymbol{\mu}+$ D.E. & Rango & $\boldsymbol{\mu}+$ E. E. & $\mu+$ D. E. & rango & $\boldsymbol{\mu}+$ D. E. & rango & $\boldsymbol{\mu}+$ D. E. & rango & $\mu+$ E. E. & $\mu+$ E. E. & $\mu+$ E. E. \\
\hline 1,0 & $80,0 \pm 4,1$ & $24,2^{\mathrm{b}}$ & $73,7 \pm 9,2^{b}$ & $61,2 \pm 13,8$ & $20,9^{\mathrm{b}}$ & $57,5 \pm 15,5$ & $20,5^{\mathrm{b}}$ & $50,0 \pm 7,1$ & $22,1^{\mathrm{b}}$ & $63,7 \pm 10,1^{\mathrm{b}}$ & $67,5 \pm 9,9^{b}$ & $67,5 \pm 8,0^{\mathrm{b}}$ \\
\hline 0,1 & $72,5 \pm 11,9$ & $18,7^{\mathrm{b}}$ & $52,5 \pm 9,2^{\mathrm{b}}$ & $53,7 \pm 21,4$ & $17,2^{\mathrm{b}}$ & $55,0 \pm 9,1$ & $20,7^{\mathrm{b}}$ & $32,5 \pm 24,7$ & $13,9^{\mathrm{ab}}$ & $56,2 \pm 10,1^{b}$ & $86,2 \pm 9,9^{b}$ & $72,5 \pm 8,0^{\mathrm{b}}$ \\
\hline 0,01 & $73,7 \pm 20,6$ & $19,2^{\mathrm{b}}$ & $63,7 \pm 9,2^{\mathrm{b}}$ & $58,7 \pm 20,2$ & $19,7^{\mathrm{b}}$ & $55,0 \pm 21,2$ & $19,0^{\mathrm{b}}$ & $50,0 \pm 22,7$ & $20,2^{\mathrm{b}}$ & $71,2 \pm 10,1^{\mathrm{b}}$ & $70,0 \pm 9,9^{b}$ & $76,2 \pm 8,0^{\mathrm{b}}$ \\
\hline 0,001 & $58,7 \pm 14,9$ & $11,9^{\mathrm{ab}}$ & $51,2 \pm 9,2^{\mathrm{b}}$ & $45,0 \pm 20,0$ & $13,7^{\mathrm{ab}}$ & $45,0 \pm 26,8$ & $13,2^{\mathrm{ab}}$ & $41,2 \pm 15,5$ & $17,2^{\mathrm{b}}$ & $67,5 \pm 10,1^{\mathrm{b}}$ & $80,0 \pm 9,9^{b}$ & $76,2 \pm 8,0^{\mathrm{b}}$ \\
\hline 0,0001 & $78,7 \pm 17,5$ & $22,0^{\mathrm{b}}$ & $75,0 \pm 9,2^{b}$ & $68,7 \pm 19,3$ & $22,7^{\mathrm{b}}$ & $58,7 \pm 21,0$ & $20,6^{\mathrm{b}}$ & $47,5 \pm 18,9$ & $17,2^{\mathrm{b}}$ & $57,5 \pm 10,1^{\mathrm{b}}$ & $68,7 \pm 9,9^{b}$ & $63,7 \pm 8,0^{\mathrm{b}}$ \\
\hline 0,00001 & $70,0 \pm 7,1$ & $17,5^{\mathrm{b}}$ & $57,5 \pm 9,2^{\mathrm{b}}$ & $47,5 \pm 14,4$ & $14,5^{\mathrm{ab}}$ & $47,5 \pm 11,9$ & $17,2^{\mathrm{b}}$ & $42,5 \pm 5,0$ & $16,1^{\mathrm{ab}}$ & $51,2 \pm 10,1^{\mathrm{ab}}$ & $73,7 \pm 9,9^{b}$ & $62,5 \pm 8,0^{\mathrm{b}}$ \\
\hline 0,000001 & $67,5 \pm 21,8$ & $15,9^{\mathrm{b}}$ & $55,0 \pm 9,2^{\mathrm{b}}$ & $63,7 \pm 29,5$ & $20,6^{\mathrm{b}}$ & $57,5 \pm 34,8$ & $18,1^{b}$ & $55,0 \pm 18,3$ & $22,0^{\mathrm{b}}$ & $65,0 \pm 10,1^{\mathrm{b}}$ & $96,2 \pm 9,9^{b}$ & $83,7 \pm 8,0^{b}$ \\
\hline 0,0 (agua) & $6,2 \pm 9,5$ & $2,5^{\mathrm{a}}$ & $5,0 \pm 9,2^{\mathrm{a}}$ & $10,0 \pm 4,1$ & $2,5^{\mathrm{a}}$ & $5,0 \pm 5,8$ & $2,5^{\mathrm{a}}$ & $7,5 \pm 6,4$ & $3,1^{\mathrm{a}}$ & $5,0 \pm 10,1^{\mathrm{a}}$ & $5,0 \pm 9,9^{\mathrm{a}}$ & $3,7 \pm 8,0^{\mathrm{a}}$ \\
\hline
\end{tabular}

$*_{\mu} \mu$ Media. D. E. = Desviación estándar. E. E. = Error experimental. Letras iguales en la misma columna no difieren estadísticamente. 
Tabla 2. Repelencia (\%) de adultos de mosca blanca Trialeurodes vaporariorum mediante extracto etanólico de clavo Syzygium aromaticum a concentraciones de 1,0 a $0,000001 \%$.

\begin{tabular}{|c|c|c|c|c|c|c|c|c|c|c|c|c|}
\hline \multirow{2}{*}{$\begin{array}{c}\text { Tiempo } \\
\text { Conc. }(\%)\end{array}$} & \multirow{2}{*}{$\frac{3.5}{{ }^{*} \mu+\text { E. E. }}$} & \multirow{2}{*}{$\frac{4}{* \mu+\text { E. E. }}$} & \multirow{2}{*}{$\begin{array}{c}5 \\
{ }^{*} \boldsymbol{\mu}+\mathbf{E} . \mathbf{E} .\end{array}$} & \multirow{2}{*}{$\frac{6}{{ }^{*} \mu+\text { E. E. }}$} & \multicolumn{2}{|c|}{12} & \multicolumn{2}{|c|}{24} & \multirow[b]{2}{*}{${ }^{*} \boldsymbol{\mu}+$ D. E. } & \multirow[b]{2}{*}{ rango } & \multicolumn{2}{|c|}{$72 \mathrm{~h}$} \\
\hline & & & & & ${ }^{*} \boldsymbol{\mu}+$ D. E. & rango & ${ }^{*} \boldsymbol{\mu}+$ D. E. & rango & & & ${ }^{*} \boldsymbol{\mu}+$ D. E. & rango \\
\hline 1,0 & $90,0 \pm 5,5^{\mathrm{c}}$ & $88,7 \pm 6,1^{\mathrm{c}}$ & $85,0 \pm 5,8^{\mathrm{d}}$ & $78,7 \pm 8,6^{\mathrm{c}}$ & $68,7 \pm 34,2$ & $23,6^{\mathrm{c}}$ & $82,5 \pm 18,5$ & $28,7^{\mathrm{c}}$ & $92,5 \pm 15,0$ & $28,2^{\mathrm{c}}$ & $100,0 \pm 0,0$ & $26,0^{\mathrm{b}}$ \\
\hline 0,1 & $77,5 \pm 5,5^{\mathrm{bc}}$ & $73,7 \pm 6,1^{\mathrm{bc}}$ & $71,2 \pm 5,8^{\mathrm{bcd}}$ & $58,7 \pm 8,6^{\mathrm{bc}}$ & $43,7 \pm 25,9$ & $19,2^{\mathrm{bc}}$ & $45,0 \pm 31,1$ & $17,1^{\mathrm{bc}}$ & $66,2 \pm 38,2$ & $19,7^{\mathrm{bc}}$ & $83,7 \pm 23,6$ & $18,4^{\mathrm{b}}$ \\
\hline 0,01 & $72,5 \pm 5,5^{\mathrm{bc}}$ & $72,5 \pm 6,1^{\mathrm{bc}}$ & $68,7 \pm 5,8^{\mathrm{bcd}}$ & $52,5 \pm 8,6^{\mathrm{bc}}$ & $35,0 \pm 16,8$ & $17,1^{\mathrm{bc}}$ & $41,2 \pm 13,8$ & $17,5^{\mathrm{bc}}$ & $46,2 \pm 38,2$ & $14,2^{\mathrm{ab}}$ & $72,5 \pm 35,7$ & $16,6^{\mathrm{ab}}$ \\
\hline 0,001 & $56,2 \pm 5,5^{b}$ & $57,5 \pm 6,1^{b}$ & $46,2 \pm 5,8^{b}$ & $37,5 \pm 8,6^{\mathrm{ab}}$ & $17,5 \pm 12,5$ & $8,7^{\text {ab }}$ & $31,2 \pm 4,8$ & $13,2^{\mathrm{ab}}$ & $47,5 \pm 23,3$ & $13,9^{\mathrm{ab}}$ & $87,5 \pm 6,4$ & $15,5^{\mathrm{b}}$ \\
\hline 0,0001 & $80,0 \pm 5,5^{\mathrm{bc}}$ & $71,2 \pm 6,1^{\mathrm{bc}}$ & $80,0 \pm 5,8^{\mathrm{cd}}$ & $71,2 \pm 8,6^{\mathrm{bc}}$ & $48,7 \pm 18,9$ & $21,6^{\mathrm{bc}}$ & $50,0 \pm 37,2$ & $19,4^{\mathrm{bc}}$ & $51,2 \pm 37,0$ & $16,0^{\mathrm{abc}}$ & $78,7 \pm 36,1$ & $18,5^{\mathrm{b}}$ \\
\hline 0,00001 & $71,2 \pm 5,5^{\mathrm{bc}}$ & $61,2 \pm 6,1^{\mathrm{bc}}$ & $57,5 \pm 5,8^{\mathrm{bc}}$ & $51,2 \pm 8,6^{\mathrm{bc}}$ & $33,7 \pm 13,1$ & $15,7^{\mathrm{abc}}$ & $37,5 \pm 12,6$ & $16,4^{\mathrm{abc}}$ & $68,7 \pm 22,5$ & $20,1^{\mathrm{bc}}$ & $85,0 \pm 17,3$ & $18,2^{\mathrm{b}}$ \\
\hline 0,000001 & $77,5 \pm 5,5^{\mathrm{bc}}$ & $76,2 \pm 6,1^{\mathrm{bc}}$ & $62,5 \pm 5,8^{\text {bcd }}$ & $66,2 \pm 8,6^{\text {bc }}$ & $43,7 \pm 22,9$ & $20,2^{\mathrm{bc}}$ & $38,7 \pm 34,7$ & $15,6^{\mathrm{ab}}$ & $53,7 \pm 25,0$ & $16,5^{\mathrm{bc}}$ & $82,5 \pm 20,2$ & $16,2^{\mathrm{b}}$ \\
\hline 0,0 (agua) & $8,7 \pm 5,5^{\mathrm{a}}$ & $10,0 \pm 6,1^{\mathrm{a}}$ & $2,5 \pm 5,8^{\mathrm{a}}$ & $7,5 \pm 8,6^{a}$ & $7,5 \pm 50$ & $3,6^{\mathrm{a}}$ & $2,5 \pm 2,9$ & $4,0^{\mathrm{a}}$ & $3,7 \pm 4,8$ & $3,2^{\mathrm{a}}$ & $6,2 \pm 2,5$ & $25^{\mathrm{a}}$ \\
\hline
\end{tabular}

* $\mu$ = Media. D. E. = Desviación estándar. E. E.= Error experimental. Letras iguales en la misma columna no difieren estadísticamente.

$84,27 \%$ de repelencia de la mosca blanca. Dado lo anterior, el mayor efecto se presentó con la concentración de $1,0 \%$ a las $72 \mathrm{~h}$ (repelencia total); sin embargo, la concentración más baja $(0,000001 \%)$ se considera efectiva porque causó de 38,7 a $82,5 \%$ de repelencia. En la Tabla 3, se observa que el extracto crudo en diclorometano de clavo causó alta repelencia de adultos de mosca blanca desde el inicio del experimento y se mantuvo a través del tiempo; a las $3 \mathrm{~h}$ la repelencia promedio de las siete concentraciones alcanzó $89,1 \%$ y a las $72 \mathrm{~h}$ fue $82,9 \%$. De acuerdo al análisis de rangos, la concentración de $0,000001 \%$ es más efectiva por repeler de 61,2 a $87,5 \%$ de adultos de mosca blanca, considerando que al utilizar bajas concentraciones de extracto la presión de selección en la mosca blanca es relativamente baja.

En el extracto crudo en hexano de clavo la repelencia promedio a las 3,5 y $72 \mathrm{~h}$ después de la aplicación de las siete concentraciones alcanzó 84,3 y $86,9 \%$, lo que indica que es mayor al $50 \%$ y se mantiene a través del tiempo, excepto a las $12 \mathrm{~h}$, con la concentración de $0,00001 \%$ (Tabla 4). Aunque, las concentraciones 1,0 y $0,01 \%$ logran mayor repelencia, la concentración más baja, de $0,000001 \%$ se considera más efectiva ya que causa de 51,2 a $87,5 \%$ de repelencia de adultos de mosca blanca. Los extractos crudos en etanol y diclorometano de clavo alcanzaron $100 \%$ de repelencia a las 72 y $48 \mathrm{~h}$, respectivamente. El mayor rango de repelencia se obtuvo con el extracto en diclorometano y de manera general se conserva el efecto de repelencia de las 3 a las $72 \mathrm{~h}$, no se biodegrada y la concentración más baja repele por lo menos una tercera parte de la población.

Se puede optar por usar el extracto crudo etanólico por su disponibilidad, economía y aceptación por las normas de agricultura orgánica. Los compuestos que repelen a la mosca blanca tienen diferente polaridad y pueden ser desde los más biodegradables que se obtienen con el etanol, polaridad más alta (García 2002), hasta los más persistentes que se extraen con el hexano.

Extractos crudos de pimienta. La repelencia provocada por el extracto en metanol de pimienta a concentraciones de 1,0 a $0,000001 \%$ en mosca blanca, va de 26,2 a $77,5 \%$ de las 3 a 72 h (Tabla 5), con el mayor efecto al 1,0\%. Esta repelencia se mantiene al aumentar el tiempo de muestreo, ya

Tabla 3. Repelencia (\%) de adultos de mosca blanca Trialeurodes vaporariorum mediante extracto diclorometanólico de clavo Syzygium aromaticum a concentraciones de 1,0 a $0,000001 \%$.

\begin{tabular}{|c|c|c|c|c|c|c|c|c|c|c|c|c|}
\hline Tiempo & 3 & 4 & 5 & 6 & 12 & & 24 & & 48 & & $72 \mathrm{~h}$ & \\
\hline Conc. (\%) & ${ }^{*} \boldsymbol{\mu}+$ E. E. & ${ }^{*} \boldsymbol{\mu}+$ E. E. & ${ }^{*} \boldsymbol{\mu}+$ E. E. & ${ }^{*} \boldsymbol{\mu}+$ E. E. & ${ }^{*} \boldsymbol{\mu}+$ D. E. & rango & ${ }^{*} \boldsymbol{\mu}+$ D. E. & rango & ${ }^{*} \boldsymbol{\mu}+$ D. E. & rango & ${ }^{*} \boldsymbol{\mu}+$ D. E. & rango \\
\hline 1,0 & $95,0 \pm 2,9^{\mathrm{bc}}$ & $97,5 \pm 3,9^{\mathrm{b}}$ & $93,7 \pm 5,0^{b}$ & $91,2 \pm 5,7^{\mathrm{b}}$ & $81,2 \pm 19,3$ & $23,7^{\mathrm{b}}$ & $85,0 \pm 12,9$ & $21,6^{\mathrm{b}}$ & $93,7 \pm 7,5$ & $21,0^{\mathrm{bc}}$ & $88,7 \pm 11,1$ & $21,6^{\mathrm{bc}}$ \\
\hline 0,1 & $92,5 \pm 2,9^{\mathrm{bc}}$ & $91,2 \pm 3,9^{\mathrm{b}}$ & $83,7 \pm 5,0^{\mathrm{b}}$ & $82,5 \pm 5,7^{\mathrm{b}}$ & $80,0 \pm 17,8$ & $23,1^{\mathrm{b}}$ & $88,7 \pm 6,3$ & $24,0^{\mathrm{b}}$ & $100 \pm 0,0$ & $275^{\mathrm{c}}$ & $93,7 \pm 2,5$ & $25,7^{\mathrm{c}}$ \\
\hline 0,01 & $91,2 \pm 2,9^{\mathrm{bc}}$ & $87,5 \pm 3,9^{b}$ & $87,5 \pm 5,0^{b}$ & $83,7 \pm 5,7^{b}$ & $72,5 \pm 24,0$ & $19,0^{\mathrm{b}}$ & $82,5 \pm 17,6$ & $19,9^{\mathrm{b}}$ & $83,7 \pm 20,2$ & $16,2^{\mathrm{bc}}$ & $82,5 \pm 18,5$ & $18,4^{\mathrm{bc}}$ \\
\hline 0,001 & $81,2 \pm 2,9^{b}$ & $82,5 \pm 3,9^{b}$ & $86,2 \pm 5,0^{b}$ & $82,5 \pm 5,7^{\mathrm{b}}$ & $57,5 \pm 8,7$ & $12,7^{\mathrm{ab}}$ & $76,2 \pm 14,4$ & $15,5^{\mathrm{ab}}$ & $92,5 \pm 6,4$ & $19,4^{\text {bc }}$ & $85,0 \pm 9,1$ & $18,2^{\mathrm{bc}}$ \\
\hline 0,0001 & $85,0 \pm 2,9^{\mathrm{bc}}$ & $81,2 \pm 3,9^{\mathrm{b}}$ & $81,2 \pm 5,0^{b}$ & $73,7 \pm 5,7^{\mathrm{b}}$ & $68,7 \pm 16,5$ & $18,9^{\mathrm{b}}$ & $73,7 \pm 20,2$ & $15,7^{\mathrm{b}}$ & $88,7 \pm 14,4$ & $18,1^{\mathrm{bc}}$ & $81,2 \pm 14,9$ & $17,4^{\text {bc }}$ \\
\hline 0,00001 & $91,2 \pm 2,9^{\mathrm{bc}}$ & $81,2 \pm 3,9^{b}$ & $80,0 \pm 5,0^{b}$ & $77,5 \pm 5,7^{\mathrm{b}}$ & $66,2 \pm 8,5$ & $17,5^{\mathrm{b}}$ & $80,0 \pm 8,2$ & $18,4^{\mathrm{b}}$ & $90,0 \pm 4,1$ & $16,9^{\mathrm{bc}}$ & $83,7 \pm 4,8$ & $17,4^{\text {bc }}$ \\
\hline 0,000001 & $87,5 \pm 2,9^{\mathrm{bc}}$ & $85,0 \pm 3,9^{b}$ & $77,5 \pm 5,0^{b}$ & $72,5 \pm 5,7^{\mathrm{b}}$ & $61,2 \pm 10,3$ & $14,5^{\mathrm{ab}}$ & $73,7 \pm 4,8$ & $14,4^{\mathrm{ab}}$ & $82,5 \pm 5,0$ & $10,4^{\mathrm{ab}}$ & $76,2 \pm 2,5$ & $10,7^{\mathrm{ab}}$ \\
\hline 0,0 (agua) & $5,0 \pm 2,9^{\mathrm{a}}$ & $5,0 \pm 3,9^{\mathrm{a}}$ & $5,0 \pm 5,0^{\mathrm{a}}$ & $2,5 \pm 5,7^{\mathrm{a}}$ & $2,5 \pm 2,9$ & $2,5^{\mathrm{a}}$ & $2,5 \pm 2,5$ & $2,5^{\mathrm{a}}$ & $2,5 \pm 2,9$ & $2,5^{\mathrm{a}}$ & $3,7 \pm 2,5$ & $2,5^{\mathrm{a}}$ \\
\hline
\end{tabular}

$*_{\mu}=$ Media. D. E. = Desviación estándar. E. E.= Error experimental. Letras iguales en la misma columna no difieren estadísticamente. 
Tabla 4. Repelencia (\%) de adultos de mosca blanca Trialeurodes vaporariorum mediante extracto hexánico de clavo Syzygium aromaticum a concentraciones de 1,0 a $0,000001 \%$.

\begin{tabular}{|c|c|c|c|c|c|c|c|c|c|c|c|}
\hline Tiempo & 3.5 & 4 & & 5 & & 6 & 12 & & 24 & 48 & $72 \mathrm{~h}$ \\
\hline Conc. (\%) & $\boldsymbol{\mu}+\mathbf{E} . \mathbf{E}$. & ${ }^{*} \boldsymbol{\mu}+$ D.E. & rango & ${ }^{*} \boldsymbol{\mu}+$ D.E. & rango & $\mu+$ E.E. & ${ }^{*} \boldsymbol{\mu}+$ D.E. & rango & $\mu+$ E.E. & $\mu+$ E.E. & $\boldsymbol{\mu}+$ E.E. \\
\hline 1 & $93,7 \pm 4,2^{\mathrm{b}}$ & $87,5 \pm 15,5$ & $23,1^{\mathrm{b}}$ & $90,0 \pm 13,5$ & $25,6^{\mathrm{c}}$ & $91,2 \pm 4,7^{\mathrm{d}}$ & $82,5 \pm 17,6$ & $26,2^{\mathrm{d}}$ & $90,0 \pm 3,4^{b}$ & $92,5 \pm 7,0^{\mathrm{b}}$ & $88,7 \pm 5,8^{b}$ \\
\hline 0,1 & $85,0 \pm 4,2^{b}$ & $82,5 \pm 11,9$ & $19,4^{\mathrm{b}}$ & $80,0 \pm 9,1$ & $18,9^{\mathrm{bc}}$ & $80,0 \pm 4,7^{\mathrm{cd}}$ & $72,5 \pm 6,4$ & $23,2^{\mathrm{cd}}$ & $83,7 \pm 3,4^{b}$ & $81,2 \pm 7,0^{\mathrm{b}}$ & $83,7 \pm 5,8^{b}$ \\
\hline 0,01 & $90,0 \pm 4,2^{\mathrm{b}}$ & $87,5 \pm 2,9$ & $22,7^{\mathrm{b}}$ & $80,0 \pm 8,2$ & $18,9^{\mathrm{bc}}$ & $77,5 \pm 4,7^{\mathrm{cd}}$ & $83,7 \pm 7,5$ & $27,2^{\mathrm{d}}$ & $96,2 \pm 3,4^{b}$ & $91,2 \pm 7,0^{b}$ & $92,5 \pm 5,8^{b}$ \\
\hline 0,001 & $83,7 \pm 4,2^{b}$ & $83,7 \pm 11,1$ & $20,1^{\mathrm{b}}$ & $81,2 \pm 7,5$ & $19,9^{\mathrm{bc}}$ & $65,5 \pm 4,7^{\mathrm{bc}}$ & $58,7 \pm 21,7$ & $15,6^{\mathrm{bcd}}$ & $90,0 \pm 3,4^{b}$ & $85,0 \pm 7,0^{\mathrm{b}}$ & $88,7 \pm 5,8^{\mathrm{b}}$ \\
\hline 0,0001 & $75,0 \pm 4,2^{\mathrm{b}}$ & $77,5 \pm 5,0$ & $14,5^{\mathrm{ab}}$ & $75,0 \pm 10,8$ & $16,0^{\mathrm{bc}}$ & $72,5 \pm 4,7^{\mathrm{bcd}}$ & $58,7 \pm 63$ & $16,0^{\mathrm{bcd}}$ & $85,0 \pm 3,4^{b}$ & $78,7 \pm 7,0^{\mathrm{b}}$ & $82,5 \pm 5,8^{b}$ \\
\hline 0,00001 & $82,5 \pm 4,2^{b}$ & $75,0 \pm 9,1$ & $14,5^{\mathrm{ab}}$ & $66,2 \pm 16,0$ & $12,2^{\mathrm{ab}}$ & $52,5 \pm 4,7^{\mathrm{b}}$ & $40,0 \pm 10,8$ & $8,2^{\mathrm{ab}}$ & $83,7 \pm 3,4^{b}$ & $68,7 \pm 7,0^{\mathrm{b}}$ & $85,0 \pm 5,8^{b}$ \\
\hline 0,000001 & $80,0 \pm 4,2^{b}$ & $78,7 \pm 13,8$ & $16,5^{\mathrm{b}}$ & $78,7 \pm 6,3$ & $18,0^{\mathrm{bc}}$ & $65,0 \pm 4,7^{\mathrm{bc}}$ & $51,2 \pm 11,1$ & $12,9^{\mathrm{abc}}$ & $81,2 \pm 3,4^{b}$ & $77,5 \pm 7,0^{\mathrm{b}}$ & $87,5 \pm 5,8^{b}$ \\
\hline 0,0 (agua) & $8,7 \pm 4,2^{\mathrm{a}}$ & $8,7 \pm 6,3$ & $2,5^{\mathrm{a}}$ & $2,5 \pm 6,3$ & $2,5^{\mathrm{a}}$ & $2,5 \pm 4,7^{\mathrm{a}}$ & $5,0 \pm 0,0$ & $2,5^{\mathrm{a}}$ & $5,0 \pm 3,4^{\mathrm{a}}$ & $5,0 \pm 7,0^{\mathrm{a}}$ & $5,0 \pm 5,8^{\mathrm{a}}$ \\
\hline
\end{tabular}

$* \mu=$ Media. D. E. = Desviación estándar. E. E.= Error experimental. Letras iguales en la misma columna no difieren estadísticamente.

que a las 3 y $72 \mathrm{~h}$ presentaron en promedio 66,2 y $63,7 \%$. De acuerdo al análisis estadístico por tiempo de muestreo las repelencias de las siete concentraciones del extracto en metanol son iguales. La concentración de $0,000001 \%$ se considera más efectiva ya que se alcanza de 40,0 a $60,0 \%$ de repelencia. El extracto crudo en etanol de pimienta a las concentraciones de 1,0 a $0,000001 \%$ causó de 17,5 a $100 \%$ de repelencia de mosca blanca (Tabla 6); las concentraciones de 0,01 y $0,00001 \%$ alcanzan $100 \%$ de repelencia a las $72 \mathrm{~h}$ de exposición, resultado importante desde el punto de vista de manejo de la mosca blanca, ya que a concentraciones bajas la repelencia se mantiene e incrementan su efecto a través del tiempo. Además, la repelencia promedio de las siete concentraciones de extracto se mantiene, a las 3 y $72 \mathrm{~h}$ se obtienen 73,5 y $89,3 \%$; se alcanza repelencia total con las concentraciones de 0,01 y $0,00001 \%$; sin embargo, la concentración más efectiva es $0,000001 \%$ por repeler de 46,2 a $87,5 \%$ de adultos de mosca blanca.

La aplicación del extracto crudo en diclorometano de pimienta a las concentraciones de 1.0 a $0,000001 \%$ en adultos de mosca blanca causó de 45,0 a $100 \%$ de repelencia de las
3 a las $72 \mathrm{~h}$ (Tabla 7). Los mayores porcentajes de repelencia se obtienen con la concentración de $1,0 \%$ que alcanza repelencia total a las 3 y $72 \mathrm{~h}$. Con la aplicación de este extracto, la repelencia se mantiene a través del tiempo de muestreo, ya que a las 3 y $72 \mathrm{~h}$ se obtienen en promedio 85,5 y $80,1 \%$. Sin embargo, la concentración de $0,000001 \%$ se considera más efectiva, ya que repele de 45,0 a $68,7 \%$, con promedio mayor al $50 \%$ de repelencia de la mosca blanca. El extracto crudo en hexano de pimienta a las concentraciones de 1,0 a $0,000001 \%$ causa de 51,2 a $100 \%$ de repelencia de adultos de mosca blanca de las 3 a 72 h, como se observa en la Tabla 8. En general el efecto repelente de todas las concentraciones se mantiene a través del tiempo de muestreo. No obstante que se alcanza la repelencia total con las concentraciones de 1,0 y 0,1 a las 4,48 y $72 \mathrm{~h}$, la concentración de $0,000001 \%$ fue más efectiva por repeler de 51,2 a $78,7 \%$ de adultos de mosca blanca.

Las concentraciones de 0,01 y $0,00001,1,0$, y $0,1 \%$ de los extractos en etanol, diclorometano y hexano de pimienta, alcanzaron $100 \%$ de repelencia de la mosca blanca. Con el primer extracto las dos concentraciones lograron esta repelencia a las $72 \mathrm{~h}$, con el segundo a las 3 y $72 \mathrm{~h}$, el tercero a las 4, 48 y

Tabla 5. Repelencia (\%) de adultos de mosca blanca Trialeurodes vaporariorum mediante extracto metanólico de pimienta Pimenta dioica a concentraciones de 1,0 a $0,000001 \%$

\begin{tabular}{|c|c|c|c|c|c|c|c|c|c|c|c|c|c|}
\hline \multirow{2}{*}{$\begin{array}{c}\text { Tiempo } \\
\text { Conc. (\%) }\end{array}$} & \multirow{2}{*}{$\begin{array}{c}3 \\
\mu+\text { E. E. }\end{array}$} & \multirow{2}{*}{$\begin{array}{c}4 \\
\mu+\text { E. E. }\end{array}$} & \multirow{2}{*}{$\begin{array}{c}5 \\
\mu+\text { E. E. }\end{array}$} & \multicolumn{2}{|l|}{6} & \multicolumn{2}{|c|}{12} & \multicolumn{2}{|l|}{24} & \multicolumn{2}{|c|}{48} & \multicolumn{2}{|c|}{$72 \mathrm{~h}$} \\
\hline & & & & $\mu+$ D. E. & rango & $\boldsymbol{\mu}+$ D. E. & rango & $\boldsymbol{\mu}+$ D. E. & rango & $\boldsymbol{\mu}+$ D. E. & rango & $\mu+$ D. E. & rango \\
\hline 1,0 & $77,5 \pm 7,3 b$ & $67,5 \pm 8,5 b$ & $65,0 \pm 7,2 b$ & $60,0 \pm 20,8$ & $23,9 b$ & $56,2 \pm 2,5$ & $25,6 \mathrm{c}$ & $57,5 \pm 8,7$ & $18,5 b$ & $75,0 \pm 7,1$ & $24,0 \mathrm{~b}$ & $72,5 \pm 11,9$ & $23,6 b$ \\
\hline 0,1 & $50,0 \pm 7,3 b$ & $41,2 \pm 8,5 \mathrm{ab}$ & $37,5 \pm 7,2 b$ & $40,0 \pm 13,5$ & $13,0 \mathrm{ab}$ & $38,7 \pm 16,5$ & $17,5 \mathrm{bc}$ & $71,2 \pm 14,4$ & $25,4 b$ & $68,7 \pm 6,3$ & $19,6 \mathrm{~b}$ & $68,7 \pm 7,5$ & $21,4 b$ \\
\hline 0,01 & $67,5 \pm 73 b$ & $43,7 \pm 8,5 b$ & $40,0 \pm 7,2 b$ & $42,5 \pm 15,0$ & $15,7 b$ & $26,2 \pm 9,5$ & $10,0 \mathrm{ab}$ & $56,2 \pm 16,5$ & $16,2 b$ & $61,2 \pm 22,9$ & $17,7 b$ & $57,5 \pm 20,6$ & $16,1 b$ \\
\hline 0,001 & $70,0 \pm 7,3 b$ & $45,0 \pm 8,5 b$ & $47,5 \pm 7,2 b$ & $47,5 \pm 6,4$ & $19,4 b$ & $41,2 \pm 13,1$ & $19,1 b c$ & $60,0 \pm 14,1$ & $18,9 b$ & $71,2 \pm 8,5$ & $21,6 b$ & $71,2 \pm 4,8$ & $24,0 \mathrm{~b}$ \\
\hline 0,0001 & $75,0 \pm 7,3 b$ & $68,7 \pm 8,5 b$ & $58,7 \pm 7,2 \mathrm{~b}$ & $50,0 \pm 14,1$ & $19,9 b$ & $41,2 \pm 18,0$ & $18,6 \mathrm{bc}$ & $57,5 \pm 6,4$ & $19,2 b$ & $62,5 \pm 20,2$ & $18,4 b$ & $62,5 \pm 9,6$ & $17,5 b$ \\
\hline 0,00001 & $58,7 \pm 7,3 b$ & $53,7 \pm 8,5 b$ & $46,2 \pm 7,2 b$ & $43,7 \pm 2,5$ & $16,9 b$ & $46,2 \pm 16,0$ & $21,6 b c$ & $52,5 \pm 5,0$ & $13,9 \mathrm{ab}$ & $51,2 \pm 23,2$ & $13,6 a b$ & $53,7 \pm 8,5$ & $11,5 \mathrm{ab}$ \\
\hline 0,000001 & $65,0 \pm 7,3 b$ & $60,0 \pm 8,5 b$ & $50,0 \pm 7,2 b$ & $50,0 \pm 12,2$ & $20,7 b$ & $40,0 \pm 14,7$ & $17,0 \mathrm{bc}$ & $55,0 \pm 4,1$ & $17,4 b$ & $60,0 \pm 0,0$ & $14,5 \mathrm{ab}$ & $60,0 \pm 10,8$ & $15,4 \mathrm{ab}$ \\
\hline 0,0 (agua) & $8,7 \pm 7,3 \mathrm{a}$ & $2,5 \pm 8,5 \mathrm{a}$ & $2,5 \pm 7,2 \mathrm{a}$ & $2,5 \pm 2,9$ & $2,5 \mathrm{a}$ & $7,5 \pm 2,9$ & $2,5 \mathrm{a}$ & $5,0 \pm 4,1$ & $2,5 \mathrm{a}$ & $10,0 \pm 7,1$ & $2,5 \mathrm{a}$ & $7,5 \pm 15,0$ & $2,5 \mathrm{a}$ \\
\hline
\end{tabular}

$* \mu=$ Media. D. E. = Desviación estándar. E. E.= Error experimental. Letras iguales en la misma columna no difieren estadísticamente. 
Tabla 6. Repelencia (\%) de adultos de mosca blanca Trialeurodes vaporariorum mediante extracto etanólico de pimienta Pimenta dioica a las concentraciones de 1,0 a $0,000001 \%$.

\begin{tabular}{|c|c|c|c|c|c|c|c|c|c|c|c|c|}
\hline \multirow{2}{*}{$\begin{array}{c}\text { Tiempo } \\
\text { Conc. (\%) }\end{array}$} & \multirow{2}{*}{$\begin{array}{c}3 \\
*_{\mu}+\text { E. E. }\end{array}$} & \multirow{2}{*}{$\begin{array}{c}4 \\
\mu+\text { E. E. }\end{array}$} & \multicolumn{2}{|c|}{5} & \multirow{2}{*}{$\frac{6}{\mu+\text { E. E. }}$} & \multicolumn{2}{|c|}{12} & \multirow{2}{*}{$\begin{array}{c}24 \\
\mu+\text { E. E. }\end{array}$} & \multicolumn{2}{|c|}{48} & \multicolumn{2}{|c|}{$72 \mathrm{~h}$} \\
\hline & & & $\mu+$ E. E. & rango & & $\mu+$ D. E. & rango & & $\mu+$ D. E. & rango & $\mu+$ D. E. & rango \\
\hline 1,0 & $88,7 \pm 5,9^{c}$ & $88,7 \pm 7,2^{\mathrm{b}}$ & $80,0 \pm 10,8$ & $28,5^{\mathrm{c}}$ & $60,0 \pm 8,1^{\mathrm{b}}$ & $45,0 \pm 23,4$ & $25,2^{\mathrm{c}}$ & $53,7 \pm 10,6^{\mathrm{b}}$ & $75,0 \pm 20,4$ & $25,5^{b}$ & $85,0 \pm 30,0$ & $18,2^{\mathrm{b}}$ \\
\hline 0,1 & $72,5 \pm 5,9^{\mathrm{bc}}$ & $68,7 \pm 7,2^{\mathrm{b}}$ & $46,2 \pm 12,5$ & $14,6^{\mathrm{ab}}$ & $52,5 \pm 8,1^{\mathrm{b}}$ & $21,2 \pm 11,1$ & $13,4^{\mathrm{abc}}$ & $17,5 \pm 10,6^{\mathrm{ab}}$ & $32,5 \pm 39,7$ & $12,6^{\mathrm{ab}}$ & $98,7 \pm 2,5$ & $19,2^{\mathrm{b}}$ \\
\hline 0,01 & $77,5 \pm 5,9^{\mathrm{bc}}$ & $65,0 \pm 7,2^{\mathrm{b}}$ & $57,5 \pm 22,2$ & $19,9^{\mathrm{bc}}$ & $63,7 \pm 8,1^{\mathrm{b}}$ & $35,0 \pm 17,3$ & $19,7^{\mathrm{bc}}$ & $37,5 \pm 10,6^{\mathrm{ab}}$ & $51,2 \pm 24,3$ & $18,2^{\mathrm{b}}$ & $100 \pm 0,0$ & $22,0^{\mathrm{b}}$ \\
\hline 0,001 & $71,2 \pm 5,9^{\mathrm{bc}}$ & $67,5 \pm 7,2^{\mathrm{b}}$ & $57,5 \pm 18,5$ & $20,0^{\mathrm{bc}}$ & $61,2 \pm 8,1^{\mathrm{b}}$ & $35,0 \pm 23,8$ & $18,0^{\mathrm{bc}}$ & $40,0 \pm 10,6^{\mathrm{ab}}$ & $55,0 \pm 38,9$ & $19,5^{\mathrm{b}}$ & $90,0 \pm 20,0$ & $18,6^{\mathrm{b}}$ \\
\hline 0,0001 & $60,0 \pm 5,9^{\mathrm{b}}$ & $58,7 \pm 7,2^{\mathrm{b}}$ & $38,7 \pm 16,0$ & $12,2^{\mathrm{ab}}$ & $40,0 \pm 8,1^{\mathrm{ab}}$ & $17,5 \pm 11,9$ & $10,4^{\mathrm{ab}}$ & $26,2 \pm 10,6^{\mathrm{ab}}$ & $33,7 \pm 13,8$ & $12,9^{\mathrm{ab}}$ & $63,7 \pm 42,3$ & $13,7^{\mathrm{ab}}$ \\
\hline 0,00001 & $67,5 \pm 5,9^{\mathrm{bc}}$ & $57,5 \pm 7,2^{\mathrm{b}}$ & $46,2 \pm 17,0$ & $14,1^{\mathrm{ab}}$ & $41,2 \pm 8,1^{b}$ & $25,0 \pm 22,7$ & $14,6^{\mathrm{abc}}$ & $31,2 \pm 10,6^{\mathrm{ab}}$ & $50,0 \pm 20,4$ & $18,2^{\mathrm{b}}$ & $100 \pm 0,0$ & $22,0^{\mathrm{b}}$ \\
\hline 0,000001 & $77,5 \pm 5,9^{\mathrm{bc}}$ & $63,7 \pm 7,2^{\mathrm{b}}$ & $60,0 \pm 21,2$ & $20,1^{\mathrm{bc}}$ & $52,5 \pm 8,1^{\mathrm{b}}$ & $48,7 \pm 16,5$ & $26,1^{\mathrm{c}}$ & $46,2 \pm 10,6^{\mathrm{ab}}$ & $62,5 \pm 14,4$ & $21,9^{b}$ & $87,5 \pm 18,9$ & $15,6^{\mathrm{b}}$ \\
\hline 0,0 (agua) & $7,5 \pm 5,9^{\mathrm{a}}$ & $3,7 \pm 7,2^{\mathrm{a}}$ & $0,0 \pm 0,0$ & $2,5 \mathrm{a}$ & $2,5 \pm 8,1^{\mathrm{a}}$ & $5,0 \pm 5,7$ & $4,5^{\mathrm{a}}$ & $3,7 \pm 10,6^{\mathrm{a}}$ & $2,5 \pm 5,0$ & $3,1^{\mathrm{a}}$ & $2,5 \pm 5,0$ & $2,5^{\mathrm{a}}$ \\
\hline
\end{tabular}

$* \mu=$ Media. D. E. = Desviación estándar. E. E.= Error experimental. Letras iguales en la misma columna no difieren estadísticamente.

$72 \mathrm{~h}$. No se tienen estudios de repelencia de mosca blanca con extractos crudos en metanol, etanol, diclorometano y hexano de hojas, flores y frutos de clavo; sin embargo, Bagavan y Rahuman (2011) aplicaron extractos en metanol y hexano de clavo al 1,0\% sobre larvas de mosquitos Anopheles vagus Dönitz, 1902, Armigeres subalbatus Coquillett, 1898 y Culex vishnui Theobald, 1901 (Diptera: Culicidae), alcanzando $100 \%$ de mortalidad. La aplicación de las concentraciones de 1,0 y 0,01, y $0,00001 \%$ de los extractos crudos en etanol de clavo y pimienta alcanzaron $100 \%$ de repelencia de la mosca blanca a las $72 \mathrm{~h}$ después del tratamiento. No se tienen evidencias del uso de extractos crudos en etanol de clavo y pimienta como repelentes de mosca blanca $T$. vaporariorum y B. tabaci. Sin embargo, se han realizado estudios de repelencia con la aplicación de extractos crudos etanólicos de otras plantas como rábano silvestre Raphanus raphanistrum L., 1753 (Brassicales: Brassicaceae), y estafiate Ambrosia artemisiifolia L., 1753 (Asterales: Asteraceae) a la concentración de $20 \%$ en mosca blanca, lograron 72 y $69 \%$ de repelencia, respectivamente (Mendoza-García et al. 2014); con extractos acuosos de comino Cuminum cyminum L., 1753 (Apiales:
Apiaceae) y tomillo Thymus vulgaris L., 1753 (Lamiales: Lamiaceae), al 4,0 \% se obtuvieron 66,1 y $62,5 \%$ de repelencia de la mosca blanca T. vaporariorum (Dehghani y Ahmadi 2013). Considerando los resultados del efecto repelente de los extractos crudos de clavo y pimienta y sus inocuos efectos en el ambiente y la salud humana, se infiere que los extractos en etanol se pueden utilizar en los programas de manejo de la mosca blanca $T$. vaporariorum, ya que alcanzan promedios de 63,3 y $57,3 \%$ de repelencia de los adultos y la concentración de $0,000001 \%$ se considera más efectiva ya que ocasiona más de 62,6 y $62,3 \%$ de repelencia; además, el etanol como disolvente se considera más seguro para su uso, tanto para el formulador y distribuidor como para el aplicador, es menos contaminante al ambiente, a los productos agrícolas cosechados y a la salud humana por su poca persistencia, al degradarse rápidamente por efecto de la temperatura y la radiación solar.

Aceites esenciales de clavo y pimienta. La repelencia de adultos de mosca blanca tratados con aceite esencial de clavo oscila de 46,2 a $100 \%$ con concentraciones de 1,0 a $0,000001 \%$ de las 3 a $72 \mathrm{~h}$ (Tabla 9); los mayores porcentajes de repelencia se

Tabla 7. Repelencia (\%) de adultos de mosca blanca Trialeurodes vaporariorum mediante extracto diclorometanólico de pimienta Pimenta dioica a las concentraciones de 1,0 a $0,000001 \%$.

\begin{tabular}{|c|c|c|c|c|c|c|c|c|c|c|c|c|c|c|c|c|}
\hline \multirow{2}{*}{$\begin{array}{c}\text { Tiempo } \\
\text { Conc. (\%) }\end{array}$} & \multicolumn{2}{|l|}{3} & \multicolumn{2}{|l|}{4} & \multicolumn{2}{|l|}{5} & \multicolumn{2}{|l|}{6} & \multicolumn{2}{|l|}{12} & \multicolumn{2}{|l|}{24} & \multicolumn{2}{|l|}{48} & \multicolumn{2}{|l|}{$72 \mathrm{~h}$} \\
\hline & $* \boldsymbol{\mu}+$ D. E. & rango & $\mu+$ D. E. & rango & $\boldsymbol{\mu}+$ D. E. & rango & $\mu+$ D. E. & rango & $\boldsymbol{\mu}+$ D. E. & rango & $\mu+$ D. E. & rango & $\mu+$ D. E. & rango & $\mu+$ D. E. & rango \\
\hline 1,0 & $100 \pm 0,0$ & $30,0^{\mathrm{d}}$ & $100 \pm 0,0$ & $29,5^{\mathrm{d}}$ & $98,7 \pm 2,5$ & $29,6^{\mathrm{c}}$ & $96,2 \pm 7,5$ & $29,0^{\mathrm{c}}$ & $95,0 \pm 7,1$ & $29,5^{\mathrm{d}}$ & $92,5 \pm 6,4$ & $27,1^{\mathrm{c}}$ & $98,7 \pm 2,5$ & $29,5^{\mathrm{d}}$ & $100 \pm 0,0$ & $27,5^{\mathrm{d}}$ \\
\hline 0,1 & $93,7 \pm 2,5$ & $23,2^{\text {cd }}$ & $92,5 \pm 2,9$ & $22,0^{\mathrm{cd}}$ & $91,2 \pm 4,8$ & $24,9^{\mathrm{c}}$ & $90,0 \pm 0,0$ & $27,0^{\mathrm{c}}$ & $90,0 \pm 0,0$ & $27,5^{\mathrm{cd}}$ & $91,2 \pm 4,8$ & $26,4^{\mathrm{c}}$ & $91,2 \pm 4,8$ & $23,1^{\mathrm{cd}}$ & $97,5 \pm 5,0$ & $25,4^{\mathrm{d}}$ \\
\hline 0,01 & $90,0 \pm 13,5$ & $21,7^{\mathrm{cd}}$ & $90,0 \pm 7,1$ & $19,7^{\mathrm{bcd}}$ & $81,2 \pm 2,5$ & $18,0^{\mathrm{bc}}$ & $80,0 \pm 8,2$ & $20,6^{\mathrm{bc}}$ & $67,5 \pm 2,9$ & $17,0^{\text {bcd }}$ & $80,0 \pm 8,2$ & $19,7^{\mathrm{bc}}$ & $86,2 \pm 7,5$ & $20,0^{\text {bed }}$ & $91,2 \pm 2,5$ & $19,7^{\mathrm{cd}}$ \\
\hline 0,001 & $90,0 \pm 4,1$ & $20,0^{\text {bed }}$ & $90,0 \pm 4,1$ & $19,6^{\text {bcd }}$ & $82,5 \pm 8,7$ & $19,5^{\mathrm{bc}}$ & $82,5 \pm 2,9$ & $21,2^{\mathrm{bc}}$ & $68,7 \pm 2,5$ & $18,5^{\mathrm{bcd}}$ & $76,2 \pm 16,5$ & $18,2^{\mathrm{bc}}$ & $75,0 \pm 14,7$ & $16,4^{\mathrm{bc}}$ & $78,7 \pm 16,5$ & $17,4^{\text {bcd }}$ \\
\hline 0,0001 & $77,5 \pm 8,7$ & $12,1^{\mathrm{abc}}$ & $90,0 \pm 10,8$ & $21,4^{\mathrm{cd}}$ & $80,0 \pm 15,8$ & $17,5^{\mathrm{bc}}$ & $66,2 \pm 2,5$ & $13,1^{\mathrm{ab}}$ & $60,0 \pm 19,1$ & $15,0^{\mathrm{abc}}$ & $72,5 \pm 17,1$ & $16,9^{\mathrm{bc}}$ & $85,0 \pm 20,4$ & $22,5^{\mathrm{cd}}$ & $86,2 \pm 21,4$ & $20,6^{\mathrm{cd}}$ \\
\hline 0,00001 & $81,2 \pm 2,5$ & $14,2^{\mathrm{abc}}$ & $70,0 \pm 0,0$ & $9,0^{\mathrm{abc}}$ & $68,7 \pm 2,5$ & $10,4^{\mathrm{ab}}$ & $65,0 \pm 0,0$ & $12,0^{\mathrm{ab}}$ & $65,0 \pm 0,0$ & $14,0^{\mathrm{ab}}$ & $58,7 \pm 2,5$ & $10,6^{\mathrm{ab}}$ & $53,7 \pm 2,5$ & $10,5^{\mathrm{ab}}$ & $62,5 \pm 2,9$ & $12,2^{\mathrm{abc}}$ \\
\hline 0,000001 & $66,2 \pm 11,1$ & $8,1^{\mathrm{ab}}$ & $68,7 \pm 8,5$ & $8,2^{\text {ab }}$ & $66,2 \pm 8,5$ & $9,6^{\mathrm{ab}}$ & $52,5 \pm 6,4$ & $6,5^{\mathrm{a}}$ & $52,5 \pm 8,7$ & $8,0^{a b}$ & $50,0 \pm 24,8$ & $10,5^{\mathrm{ab}}$ & $45,0 \pm 108$ & $7,5^{\mathrm{ab}}$ & $45,0 \pm 9,1$ & $6,6^{\mathrm{ab}}$ \\
\hline 0,0 (agua) & $6,2 \pm 2,5$ & $2,5^{\mathrm{a}}$ & $6,2 \pm 2,5$ & $2,5^{\mathrm{a}}$ & $5,0 \pm 0,0$ & $2,5^{\mathrm{a}}$ & $5,0 \pm 0,0$ & $2,5^{\mathrm{a}}$ & $10,0 \pm 0,0$ & $2,5^{\mathrm{a}}$ & $6,2 \pm 2,5$ & $2,5^{\mathrm{a}}$ & $6,2 \pm 2,5$ & $2,5^{\mathrm{a}}$ & $6,2 \pm 2,5$ & $2,5^{\mathrm{a}}$ \\
\hline
\end{tabular}

$* \mu=$ Media. D. E. $=$ Desviación estándar. E. E.= Error experimental. Letras iguales en la misma columna no difieren estadísticamente. 
Tabla 8. Repelencia (\%) de adultos de mosca blanca Trialeurodes vaporariorum mediante extracto hexánico de pimienta Pimenta dioica a las concentraciones de 1,0 a $0,000001 \%$.

\begin{tabular}{|c|c|c|c|c|c|c|c|c|c|c|c|c|c|c|c|}
\hline \multirow{2}{*}{$\begin{array}{c}\text { Tiempo } \\
\text { Conc. (\%) }\end{array}$} & \multirow{2}{*}{$\begin{array}{c}3 \\
{ }^{*} \boldsymbol{\mu}+\text { E. E. }\end{array}$} & \multicolumn{2}{|l|}{4} & \multicolumn{2}{|l|}{5} & \multicolumn{2}{|l|}{6} & \multicolumn{2}{|l|}{12} & \multicolumn{2}{|c|}{24} & \multicolumn{2}{|l|}{48} & \multicolumn{2}{|c|}{$72 \mathrm{~h}$} \\
\hline & & $\boldsymbol{\mu}+$ D. E. & rango & $\boldsymbol{\mu}+$ D. E. & rango & $\boldsymbol{\mu}+$ D. E. & rango & $\mu+$ D. E. & rango & $\boldsymbol{\mu}+$ D. E. & rango & $\boldsymbol{\mu}+$ D. E. & rango & $\boldsymbol{\mu}+$ D. E. & rango \\
\hline 1,0 & $97,5 \pm 4,1^{\mathrm{d}}$ & $96,2 \pm 4,8$ & $25,9^{\text {cd }}$ & $96,5 \pm 2,5$ & $28,9^{c}$ & $98,7 \pm 25$ & $28,7^{\mathrm{c}}$ & $97,5 \pm 2,9$ & $29,5^{\mathrm{c}}$ & $98,7 \pm 2,5$ & $29,7^{\mathrm{d}}$ & $98,7 \pm 2,5$ & $27,4^{\mathrm{c}}$ & $98,7 \pm 2,5$ & $28,0^{c}$ \\
\hline 0,1 & $96,2 \pm 4,1^{\mathrm{cd}}$ & $100 \pm 0,0$ & $29,5^{\mathrm{d}}$ & $91,2 \pm 6,3$ & $246^{\mathrm{c}}$ & $92,5 \pm 6,4$ & $23,6^{\mathrm{bc}}$ & $78,7 \pm 7,5$ & $19,7^{\mathrm{bc}}$ & $90,0 \pm 10,8$ & $24,9^{\text {cd }}$ & $100 \pm 0,0$ & $28,5^{\mathrm{c}}$ & $100 \pm 0,0$ & $29,0^{c}$ \\
\hline 0,01 & $85,0 \pm 4,1^{\text {bcd }}$ & $85,0 \pm 10,8$ & $17,7^{\mathrm{bcd}}$ & $80,0 \pm 14,1$ & $17,7^{\mathrm{bc}}$ & $80,0 \pm 16,8$ & $17,1^{\mathrm{bc}}$ & $72,5 \pm 24,0$ & $18,1^{\mathrm{bc}}$ & $76,2 \pm 15,5$ & $18,2^{\text {bcd }}$ & $80,0 \pm 9,1$ & $16,1^{\mathrm{bc}}$ & $83,7 \pm 2,5$ & $20,4^{\mathrm{bc}}$ \\
\hline 0,001 & $92,5 \pm 4,1^{\text {bed }}$ & $87,5 \pm 6,4$ & $18,6^{\mathrm{bcd}}$ & $83,7 \pm 8,5$ & $19,7^{\mathrm{bc}}$ & $83,7 \pm 13,1$ & $18,2^{\mathrm{bc}}$ & $75,0 \pm 17,8$ & $18,4^{\mathrm{bc}}$ & $78,7 \pm 6,3$ & $18,6^{\text {bed }}$ & $80,0 \pm 4,1$ & $16,5^{\mathrm{bc}}$ & $83,7 \pm 2,5$ & $20,4^{\mathrm{bc}}$ \\
\hline 0,0001 & $77,5 \pm 4,1^{\mathrm{bc}}$ & $68,7 \pm 2,5$ & $9,9^{\mathrm{ab}}$ & $77,5 \pm 11,9$ & $16,2^{\mathrm{bc}}$ & $77,5 \pm 11,9$ & $15,1^{\mathrm{ab}}$ & $68,7 \pm 2,5$ & $15,7 \mathrm{~b}$ & $67,5 \pm 2,9$ & $12,7^{\mathrm{abc}}$ & $73,7 \pm 2,5$ & $11,9^{\mathrm{ab}}$ & $73,7 \pm 2,5$ & $13,1^{\mathrm{ab}}$ \\
\hline 0,00001 & $78,7 \pm 4,1^{\text {bed }}$ & $73,7 \pm 18,9$ & $12,9^{\mathrm{abc}}$ & $65,0 \pm 17,8$ & $11,2^{\mathrm{ab}}$ & $68,7 \pm 18,9$ & $11,7^{\mathrm{ab}}$ & $58,7 \pm 29,3$ & $14,2^{\mathrm{ab}}$ & $60,0 \pm 34,6$ & $15,0^{\mathrm{abc}}$ & $85,0 \pm 12,2$ & $19,2^{\mathrm{bc}}$ & $62,5 \pm 5,0$ & $7,9^{\mathrm{ab}}$ \\
\hline 0,000001 & $76,2 \pm 4,1^{b}$ & $78,7 \pm 14,9$ & $15,0^{\mathrm{abc}}$ & $68,7 \pm 7,5$ & $11,0^{\mathrm{ab}}$ & $77,5 \pm 13,2$ & $14,9^{\mathrm{ab}}$ & $58,7 \pm 27,8$ & $13,7^{\mathrm{ab}}$ & $51,2 \pm 17,0$ & $10,2^{\mathrm{ab}}$ & $71,2 \pm 9,5$ & $9,9^{\mathrm{ab}}$ & $68,7 \pm 10,3$ & $10,7^{\mathrm{ab}}$ \\
\hline 0,0 (agua) & $7,5 \pm 4,1^{\mathrm{a}}$ & $7,5 \pm 2,9$ & $2,5^{\mathrm{a}}$ & $10,0 \pm 0,0$ & $2,5^{\mathrm{a}}$ & $0,0 \pm 0,0$ & $2,5^{\mathrm{a}}$ & $7,5 \pm 2,9$ & $2,5^{\mathrm{a}}$ & $7,5 \pm 2,9$ & $2,5^{\mathrm{a}}$ & $7,5 \pm 2,9$ & $2,5^{\mathrm{a}}$ & $6,2 \pm 4,8$ & $2,5^{\mathrm{a}}$ \\
\hline
\end{tabular}

$* \mu=$ Media. D. E. $=$ Desviación estándar. E. E.= Error experimental. Letras iguales en la misma columna no difieren estadísticamente.

obtienen al $1,0 \%$ de aceite y va de 83,7 a $96,2 \%$ de las 3 a $24 \mathrm{~h}$ y $100 \%$ de repelencia a las 48 y 72 h después de la aplicación. Es importante indicar que el efecto repelente del aceite esencial de clavo a las concentraciones de 0,000001 a $1,0 \%$ se mantienen ya que presentan 87,3 y $90,1 \%$ de repelencia promedio a las 3 y $72 \mathrm{~h}$, con un ligero incremento en el último muestreo. Sin embargo, la concentración de $0,000001 \%$ es más efectiva por repeler de 62,5 a $88,7 \%$ de los adultos de mosca blanca. El efecto repelente del aceite esencial de pimienta en mosca blanca con las concentraciones de 1,0 a $0,000001 \%$ se observa en la Tabla 10, que va de 57,5 a 97,5\% de las 3 a $72 \mathrm{~h}$. La repelencia de las siete concentraciones del aceite de pimienta se mantiene a través del tiempo, a las $3 \mathrm{~h}$ se determinó $84,2 \%$ y a las $72 \mathrm{~h}$ es de $85,9 \%$ en promedio. Estadísticamente no existen diferencias entre tratamientos y tiempos de muestreo, la concentración de $0,000001 \%$ se considera más efectiva por repeler de 63,7 a $78,7 \%$ de adultos de mosca blanca. Los aceites esenciales de clavo y pimienta al 1,0 y $0,000001 \%$ causaron de 46,2 a $100 \%$ de repelencia de mosca blanca. En la mayoría de las observaciones de las siete concentraciones, la repelencia es mayor al $50 \%$; en el muestreo de las
$12 \mathrm{~h}$, la repelencia disminuye $22,7 \%$, pero a partir de las 24 $\mathrm{h}$ el efecto repelente de ambos aceites se recupera y se mantienen, con incremento de $25,5 \%$ de repelencia a las $72 \mathrm{~h}$. El aceite esencial de clavo se considera más efectivo ya que la concentración de 1,0\% alcanza $100 \%$ de repelencia a las 48 y $72 \mathrm{~h}$, en promedio incrementa 2,6 veces más que la pimienta. No hay registros de repelencia de mosca blanca con aceites esenciales de clavo y pimienta; el aceite esencial de clavo se ha aplicado en otros insectos con efectividad variable, incluso mayores a los encontrados en este trabajo y en algunos insectos causa mortalidad. Se reporta que ocasiona $100 \%$ de repelencia en Tribolium castaneum Herbst, 1797 (Coleoptera: Tenebrionidae), la concentración de 0,2 \% (Abo-El-Saad et al. 2011), 80,3 y 60,0 \% en mosquitos Aedes aegypti L., 1762 (Diptera: Culicidae), y Culex quinquefasciatus Say, 1823 (Diptera: Culicidae), con $\mathrm{DE}_{50}$ menor a 0,045 y 0,003 $\mathrm{mg} . \mathrm{cm}^{-2}$ (Phasomkusolsil y Soonwera 2011). Con aceite esencial de pimienta aplicado a larvas de mosquito $C$. quinquefasciatus, se determinó $\mathrm{CL}_{50}$ de 7,72 \% (Pavela 2009), y en termitas obreras Reticulitermes speratus Kolbe, 1885 (Isoptera: Rhinotermitidae), a la concentración de $1 \mu \mathrm{g}$.papel ${ }^{-1}$

Tabla 9. Repelencia (\%) de adultos de mosca blanca Trialeurodes vaporariorum mediante aceite esencial de clavo Syzigium aromaticum a concentraciones de 1,0 a $0,000001 \%$.

\begin{tabular}{|c|c|c|c|c|c|c|c|c|c|c|c|c|c|}
\hline Tiempo & 3 & & 4 & & 5 & 6 & 12 & 24 & & 48 & & $72 \mathrm{~h}$ & \\
\hline Conc. $(\%)$ & ${ }^{*} \boldsymbol{\mu}+$ D. E. & rango & $\boldsymbol{\mu}+$ D. E. & rango & $\mu+$ E. E. & $\boldsymbol{\mu}+$ E. E. & $\boldsymbol{\mu}+$ E. E. & $\boldsymbol{\mu}+$ D. E. & rango & $\boldsymbol{\mu}+$ D. E. & rango & $\mu+$ D. E. & rango \\
\hline 1 & $95,0 \pm 4,1$ & $26,2^{\mathrm{c}}$ & $90,0 \pm 8,2$ & $23,7^{c}$ & $85,0 \pm 5,8^{c}$ & $85,0 \pm 5,4^{c}$ & $83,7 \pm 9,8^{b}$ & $96,2 \pm 4,8$ & $29,0^{c}$ & $100 \pm 0,0$ & $28,5^{\mathrm{c}}$ & $100 \pm 0,0$ & $26,5^{b}$ \\
\hline 0,1 & $81,2 \pm 2,5$ & $26,2^{\mathrm{c}}$ & $81,2 \pm 13,1$ & $16,4^{\mathrm{bc}}$ & $78,7 \pm 5,8^{\mathrm{bc}}$ & $80,0 \pm 5,4^{c}$ & $63,7 \pm 9,8^{b}$ & $80,0 \pm 12,2$ & $19,6^{\mathrm{bc}}$ & $90,0 \pm 16,8$ & $22,0^{\mathrm{bc}}$ & $90,0 \pm 16,8$ & $19,1^{b}$ \\
\hline 0,01 & $91,2 \pm 2,5$ & $21,0^{\mathrm{bc}}$ & $86,2 \pm 2,5$ & $21,1^{\mathrm{bc}}$ & $77,5 \pm 5,8^{\mathrm{bc}}$ & $81,2 \pm 5,4^{\mathrm{c}}$ & $56,2 \pm 9,8^{b}$ & $72,5 \pm 11,9$ & $15,7^{\mathrm{b}}$ & $82,5 \pm 9,6$ & $16,5^{\mathrm{bc}}$ & $92,5 \pm 9,6$ & $19,5^{b}$ \\
\hline 0,001 & $83,7 \pm 11,1$ & $15,6^{\mathrm{bc}}$ & $83,7 \pm 8,5$ & $18,0^{\mathrm{bc}}$ & $73,7 \pm 5,8^{\mathrm{bc}}$ & $75,0 \pm 5,4^{\mathrm{c}}$ & $58,7 \pm 9,8^{b}$ & $62,5 \pm 25,3$ & $13,4^{\mathrm{ab}}$ & $75,0 \pm 22,7$ & $15,4^{\mathrm{ab}}$ & $81,2 \pm 14,4$ & $13,6^{\mathrm{ab}}$ \\
\hline 0,0001 & $95,0 \pm 0,0$ & $27,0^{c}$ & $88,7 \pm 7,5$ & $23,0^{\mathrm{c}}$ & $81,2 \pm 5,8^{\mathrm{bc}}$ & $81,2 \pm 5,4^{\mathrm{c}}$ & $77,5 \pm 9,8^{b}$ & $80,0 \pm 21,2$ & $20,4^{b c}$ & $86,2 \pm 15,5$ & $19,2^{\mathrm{bc}}$ & $88,7 \pm 16,0$ & $17,0^{\mathrm{b}}$ \\
\hline 0,00001 & $80,0 \pm 10,8$ & $12,0^{\mathrm{ab}}$ & $75,0 \pm 4,1$ & $9,5^{\mathrm{ab}}$ & $57,5 \pm 5,8^{b}$ & $46,2 \pm 5,4^{b}$ & $50,0 \pm 9,8^{\mathrm{ab}}$ & $58,7 \pm 20,2$ & $11,5^{\mathrm{ab}}$ & $67,5 \pm 12,6$ & $10,7^{\mathrm{ab}}$ & $90,0 \pm 10,8$ & $17,1^{\mathrm{b}}$ \\
\hline 0,000001 & $85,0 \pm 10,8$ & $16,6^{\mathrm{bc}}$ & $83,7 \pm 7,5$ & $17,7^{\mathrm{bc}}$ & $81,2 \pm 5,8^{\mathrm{bc}}$ & $77,5 \pm 5,4^{\mathrm{c}}$ & $62,5 \pm 9,8^{b}$ & $81,2 \pm 12,5$ & $19,9^{\mathrm{bc}}$ & $83,7 \pm 13,1$ & $17,1^{\mathrm{bc}}$ & $88,7 \pm 13,1$ & $16,6^{b}$ \\
\hline 0,0 (agua) & $8,7 \pm 10,3$ & $2,5^{\mathrm{a}}$ & $10,0 \pm 12,2$ & $2,5^{\mathrm{a}}$ & $2,5 \pm 5,8^{\mathrm{a}}$ & $2,5 \pm 5,4^{\mathrm{a}}$ & $7,5 \pm 9,8^{\mathrm{a}}$ & $5,0 \pm 0,0$ & $2,5^{\mathrm{a}}$ & $6,2 \pm 2,5$ & $2,5^{\mathrm{a}}$ & $3,7 \pm 2,5$ & $2,5^{\mathrm{a}}$ \\
\hline
\end{tabular}

$*_{\mu}=$ Media. D. E. = Desviación estándar. E. E.= Error experimental. Letras iguales en la misma columna no difieren estadísticamente. 
Tabla 10. Repelencia (\%) de adultos de mosca blanca Trialeurodes vaporariorum mediante aceite esencial de pimienta Pimenta dioica a concentraciones de 1,0 a $0,000001 \%$.

\begin{tabular}{|c|c|c|c|c|c|c|c|c|c|c|c|c|c|}
\hline Tiempo & 3 & 4 & & 5 & & 6 & & 12 & & 24 & & 48 & $72 \mathrm{~h}$ \\
\hline Conc. (\%) & ${ }^{*} \boldsymbol{\mu}+$ E. E. & $\boldsymbol{\mu}+$ D. E. & rango & $\boldsymbol{\mu}+$ D. E. & rango & $\mu+$ D. E. & rango & $\boldsymbol{\mu}+$ D. E. & rango & $\boldsymbol{\mu}+$ D. E. & rango & $\boldsymbol{\mu}+$ E. E. & $\boldsymbol{\mu}+$ E. E. \\
\hline 1,0 & $91,2 \pm 5,9^{b}$ & $87,5 \pm 8,7$ & $23,1^{\mathrm{b}}$ & $83,7 \pm 11,1$ & $21,4^{\mathrm{b}}$ & $85,0 \pm 12,9$ & $25,7^{\mathrm{b}}$ & $76,2 \pm 16,5$ & $23,4^{\mathrm{b}}$ & $66,2 \pm 22,9$ & $16,6^{\mathrm{b}}$ & $78,7 \pm 4,5^{\mathrm{b}}$ & $88,7 \pm 5,8^{b}$ \\
\hline 0,1 & $88,7 \pm 5,9^{b}$ & $86,2 \pm 11,1$ & $24,5^{\mathrm{b}}$ & $83,7 \pm 12,5$ & $23,9^{b}$ & $73,7 \pm 17,0$ & $20,7^{b}$ & $63,7 \pm 11,1$ & $17,5^{\mathrm{b}}$ & $57,5 \pm 15,0$ & $14,4^{\mathrm{ab}}$ & $81,2 \pm 4,5^{\mathrm{b}}$ & $83,7 \pm 5,8^{b}$ \\
\hline 0,01 & $85,0 \pm 5,9^{b}$ & $72,5 \pm 6,4$ & $12,5^{\mathrm{ab}}$ & $70,0 \pm 7,1$ & $13,0^{\mathrm{ab}}$ & $62,5 \pm 15,0$ & $15,0^{\mathrm{ab}}$ & $61,2 \pm 8,5$ & $15,6^{\mathrm{b}}$ & $61,2 \pm 8,5$ & $16,0^{\mathrm{b}}$ & $87,5 \pm 4,5^{b}$ & $90,0 \pm 5,8^{b}$ \\
\hline 0,001 & $82,5 \pm 5,9^{b}$ & $81,2 \pm 14,4$ & $21,5^{\mathrm{b}}$ & $80,0 \pm 14,1$ & $21,1^{\mathrm{b}}$ & $72,5 \pm 26,0$ & $20,6^{b}$ & $73,7 \pm 20,2$ & $22,9^{\mathrm{b}}$ & $78,7 \pm 18,9$ & $24,1^{\mathrm{b}}$ & $88,7 \pm 4,5^{b}$ & $97,5 \pm 5,8^{b}$ \\
\hline 0,0001 & $80,0 \pm 5,9^{b}$ & $73,7 \pm 16,5$ & $15,9^{\mathrm{b}}$ & $72,5 \pm 15,5$ & $16,1^{\mathrm{b}}$ & $60,0 \pm 4,1$ & $13,9^{\mathrm{ab}}$ & $63,7 \pm 8,5$ & $17,0^{\mathrm{b}}$ & $71,2 \pm 10,3$ & $21,7^{\mathrm{b}}$ & $80,0 \pm 4,5^{\mathrm{b}}$ & $85,0 \pm 5,8^{b}$ \\
\hline 0,00001 & $83,7 \pm 5,9^{b}$ & $76,2 \pm 18,0$ & $18,3^{\mathrm{b}}$ & $72,5 \pm 15,0$ & $16,1^{\mathrm{b}}$ & $62,5 \pm 13,2$ & $15,2^{\mathrm{ab}}$ & $60,0 \pm 10,8$ & $14,9^{\mathrm{ab}}$ & $70,0 \pm 16,8$ & $19,6^{\mathrm{b}}$ & $85,0 \pm 4,5^{\mathrm{b}}$ & $80,0 \pm 5,8^{b}$ \\
\hline 0,000001 & $78,7 \pm 5,9^{\mathrm{b}}$ & $71,2 \pm 15,5$ & $13,9^{\mathrm{ab}}$ & $73,7 \pm 18,0$ & $17,9^{\mathrm{b}}$ & $70,0 \pm 23,4$ & $18,2^{\mathrm{b}}$ & $66,2 \pm 21,7$ & $18,2^{\mathrm{b}}$ & $63,7 \pm 19,3$ & $17,0^{\mathrm{b}}$ & $76,2 \pm 4,5^{b}$ & $76,2 \pm 5,8^{b}$ \\
\hline 0,0 (agua) & $7,5 \pm 5,9^{\mathrm{a}}$ & $5,0 \pm 0,0$ & $2,5^{\mathrm{a}}$ & $5,0 \pm 0,0$ & $2,5^{\mathrm{a}}$ & $0,0 \pm 0,0$ & $2,5^{\mathrm{a}}$ & $5,0 \pm 0,0$ & $2,5^{\mathrm{a}}$ & $5,0 \pm 0,0$ & $2,5^{\mathrm{a}}$ & $5,0 \pm 4,5^{\mathrm{a}}$ & $5,0 \pm 5,8^{\mathrm{a}}$ \\
\hline
\end{tabular}

$* \mu=$ Media. D. E. = Desviación estándar. E. E.= Error experimental. Letras iguales en la misma columna no difieren estadísticamente.

provoca $100 \%$ de mortalidad (Seon-Mi et al. 2009). En moscas Camptomyia corticalis Loew, 1851 (Diptera: Cecidomyiidae), por efecto fumigante alcanza 75,0 y 93,0 \% de mortalidad a las concentraciones de 1,0 y $1,4 \mathrm{mg} \cdot \mathrm{cm}^{-3}$ (Jun-Ran et al. 2012), y la mezcla de aceite de pimienta con aceite de palo de rosa Aniba rosaeodora Ducke, 1930 (Laurales: Lauraceae), aplicado al mosquito $A$. aegypti se determinó la $\mathrm{CL}_{50}$ de $11,4 \%$ (Schalcher et al. 2014). Se tienen reportes de repelencia de mosca blanca $T$. vaporariorum y $B$. tabaci, con aceites esenciales de otras plantas con efecto diverso. El aceite esencial de frutos del pimentero brasileño Schinus terebinthifolius Raddi, 1820 (Sapindales: Anacardiaceae), al 1,0 \% alcanza $37,8 \%$ de repelencia y con eucalipto limón Corymbia citriodora (Hook) K. D. Hill y L. A. S. Johnson, 1995 (Myrtales: Myrtaceae), se determinó la $\mathrm{CL}_{50}$ de $0,025 \%$ para la mosca blanca B. tabaci (Hussein et al. 2017). Los aceites esenciales de orégano Origanum minutiflorum O. Schwarz y P. H. Davis, 1949, lavanda Lavandula hybrida Devantville, hierbabuena Mentha piperita L., 1753 (Lamiales: Lamiaceae), eucalipto Eucalyptus globulus Labill., 1792 (Myrtales: Myrtaceae), cedro Cedrus atlantica (Endl.) Manetti ex Carrière, 1867 (Pi- nales: Pinaceae) y jengibre Zingiber officinale Roscoe, 1807 (Zingiberales: Zingiberaceae), presentan índices de repelencia de $87,14,20,25,30,09,49,37,25,39$ y 7,51 , respectivamente (Birgücü et al. 2016). El aceite esencial de geranio Pelargonium graveolens L'Hér., 1789 (Geraniales: Geraniaceae), a de 0,00005 a $0,0002 \%$ logra $70,0 \%$ de repelencia de la mosca blanca $B$. tabaci de las 3 a $24 \mathrm{~h}$ de exposición (Baldin et al. 2015), con el aceite esencial de Plectranthus neochilus Schltr, 1896 (Lamiales: Lamiaceae), al 1,0 \% alcanza $76,7 \%$ de repelencia de $B$. tabaci a las $24 \mathrm{~h}$ (Baldin et al. 2013). Los aceites esenciales de flores, hojas y planta completa de anís de monte Tagetes filifolia Lag., 1816 (Asterales: Asteraceae), a las concentraciones de 0,0001 y $10,0 \%$ alcanzan 89,3 y $100 \%$ de repelencia, con $\mathrm{CR}_{50}$ de $0,013,0,023$ y $0,024 \%$, respectivamente (Camarillo et al. 2009).

Compuestos secundarios de clavo y pimienta. La repelencia de adultos de mosca blanca T. vaporariorum con el compuesto eugenol comercial (Sigma-Aldrich) a concentraciones de 1,0 a $0,000001 \%$ de las 3 a las $72 \mathrm{~h}$ se observa en la Tabla 11 , donde se constata que va de 18,7 a $97,5 \%$; la concentración

Tabla 11. Repelencia (\%) de adultos de mosca blanca Trialeurodes vaporariorum mediante eugenol a concentraciones de 1,0 a $0,000001 \%$.

\begin{tabular}{|c|c|c|c|c|c|c|c|c|c|c|}
\hline Tiempo & & & 4 & 5 & 6 & 12 & 24 & 4 & & $72 \mathrm{~h}$ \\
\hline Conc. (\%) & $* \mu+$ D. E. & rango & $\mu+$ E. E. & $\mu+$ E. E. & $\mu+$ E. E. & $\mu+$ E. E. & $\mu+$ E. E. & $\boldsymbol{\mu}+$ D. E. & rango & $\mu+$ E. E. \\
\hline 1,0 & $77,5 \pm 12,6$ & $27,7^{\mathrm{c}}$ & $73,7 \pm 8,5^{\mathrm{c}}$ & $60,0 \pm 8,4^{\mathrm{b}}$ & $56,2 \pm 8,7^{\mathrm{b}}$ & $60,0 \pm 7,8^{\mathrm{b}}$ & $56,2 \pm 8,5^{\mathrm{b}}$ & $66,2 \pm 16,5$ & $20,9^{\mathrm{bc}}$ & $68,7 \pm 10,6^{\mathrm{b}}$ \\
\hline 0,1 & $46,2 \pm 21,4$ & $14,7^{\mathrm{abc}}$ & $50,0 \pm 8,5^{\mathrm{bc}}$ & $42,5 \pm 8,4^{\mathrm{ab}}$ & $30,0 \pm 8,7^{\mathrm{ab}}$ & $36,2 \pm 7,8^{\mathrm{ab}}$ & $32,5 \pm 8,5^{\mathrm{ab}}$ & $47,5 \pm 27,2$ & $15,1^{\mathrm{ab}}$ & $48,7 \pm 10,6^{a b}$ \\
\hline 0,01 & $58,7 \pm 21,7$ & $19,6^{\mathrm{bc}}$ & $48,7 \pm 8,5^{\mathrm{bc}}$ & $41,2 \pm 8,4^{\mathrm{ab}}$ & $36,2 \pm 8,7^{\mathrm{ab}}$ & $40,0 \pm 7,8^{\mathrm{ab}}$ & $38,7 \pm 8,5^{\mathrm{ab}}$ & $36,2 \pm 2,5$ & $9,4^{\mathrm{ab}}$ & $50,0 \pm 10,6^{\mathrm{ab}}$ \\
\hline 0,001 & $65,0 \pm 58$ & $22,2^{\mathrm{bc}}$ & $57,0 \pm 8,5^{\mathrm{bc}}$ & $55,0 \pm 8,4^{b}$ & $50,0 \pm 8,7^{\mathrm{ab}}$ & $56,2 \pm 7,8^{\mathrm{b}}$ & $60,0 \pm 8,5^{\mathrm{b}}$ & $50,0 \pm 17,8$ & $15,5^{\mathrm{ab}}$ & $71,2 \pm 10,6^{b}$ \\
\hline 0,0001 & $38,7 \pm 18,9$ & $11,6^{\mathrm{ab}}$ & $30,0 \pm 8,5^{\mathrm{ab}}$ & $27,5 \pm 8,4^{\mathrm{ab}}$ & $18,7 \pm 8,7^{\mathrm{ab}}$ & $37,5 \pm 7,8^{\mathrm{ab}}$ & $48,7 \pm 8,5^{\mathrm{b}}$ & $68,7 \pm 27,8$ & $21,9^{\mathrm{bc}}$ & $78,7 \pm 10,6^{b}$ \\
\hline 0,00001 & $46,2 \pm 10,3$ & $14,1^{\mathrm{ab}}$ & $36,2 \pm 8,5^{\mathrm{abc}}$ & $38,7 \pm 8,4^{\mathrm{ab}}$ & $31,2 \pm 8,7^{\mathrm{ab}}$ & $32,7 \pm 7,8^{\mathrm{ab}}$ & $41,2 \pm 8,5^{\mathrm{ab}}$ & $56,2 \pm 22,9$ & $17,1^{\mathrm{bc}}$ & $60,0 \pm 10,6^{\mathrm{ab}}$ \\
\hline 0,000001 & $58,7 \pm 25,9$ & $19,1^{\mathrm{bc}}$ & $48,7 \pm 8,5^{\mathrm{bc}}$ & $45,0 \pm 8,4^{\mathrm{ab}}$ & $38,7 \pm 8,7^{\mathrm{ab}}$ & $52,5 \pm 7,8^{b}$ & $71,2 \pm 8,5^{\mathrm{b}}$ & $92,5 \pm 8,7$ & $29,4^{\mathrm{c}}$ & $97,5 \pm 10,6^{\mathrm{b}}$ \\
\hline 0,0 (agua) & $11,2 \pm 4,7$ & $2,7^{\mathrm{a}}$ & $7,5 \pm 8,5^{\mathrm{a}}$ & $8,7 \pm 8,4^{a}$ & $10,0 \pm 8,7^{\mathrm{a}}$ & $8,7 \pm 7,8^{\mathrm{a}}$ & $8,7 \pm 8,5^{\mathrm{a}}$ & $7,5 \pm 2,9$ & $2,7^{\mathrm{a}}$ & $11,2 \pm 10,6^{\mathrm{a}}$ \\
\hline
\end{tabular}

$* \mu=$ Media. D. E. = Desviación estándar. E. E.= Error experimental. Letras iguales en la misma columna no difieren estadísticamente. 
Tabla 12. Repelencia (\%) de adultos de mosca blanca Trialeurodes vaporariorum mediante metil eugenol a concentraciones de 1,0 a $0,000001 \%$.

\begin{tabular}{|c|c|c|c|c|c|c|c|c|c|}
\hline Tiempo & 3 & 4 & 5 & 6 & 12 & 24 & 48 & \multicolumn{2}{|c|}{$72 \mathrm{~h}$} \\
\hline Conc. $(\%)$ & $* \boldsymbol{\mu}+$ E. E. & $\mu+$ E. E. & $\mu+$ E. E. & $\boldsymbol{\mu}+$ E. E. & $\boldsymbol{\mu}+$ E. E. & $\boldsymbol{\mu}+$ E. E. & $\boldsymbol{\mu}+$ E. E. & $\boldsymbol{\mu}+$ D. E. & rango \\
\hline 1,0 & $63,7 \pm 7,4^{\mathrm{bc}}$ & $63,7 \pm 8,3^{b}$ & $56,2 \pm 9,7^{\mathrm{b}}$ & $56,2 \pm 9,6^{\mathrm{b}}$ & $50,0 \pm 8,9^{b}$ & $47,5 \pm 9,7^{\mathrm{ab}}$ & $56,2 \pm 7,7^{\mathrm{b}}$ & $62,5 \pm 24,7$ & $16,2^{\mathrm{b}}$ \\
\hline 0,1 & $75,0 \pm 7,4^{\mathrm{c}}$ & $71,2 \pm 8,3^{b}$ & $61,2 \pm 9,7^{b}$ & $58,7 \pm 9,6^{\mathrm{b}}$ & $61,2 \pm 8,9^{b}$ & $52,5 \pm 9,7^{b}$ & $67,5 \pm 7,7^{\mathrm{b}}$ & $65,0 \pm 23,4$ & $17,1^{\mathrm{b}}$ \\
\hline 0,01 & $56,2 \pm 7,4^{\mathrm{bc}}$ & $50,0 \pm 8,3^{b}$ & $37,5 \pm 9,7^{\mathrm{ab}}$ & $38,7 \pm 9,6^{\mathrm{ab}}$ & $43,7 \pm 8,9^{\mathrm{ab}}$ & $41,2 \pm 9,7^{\mathrm{ab}}$ & $47,5 \pm 7,7^{b}$ & $58,7 \pm 14,3$ & $14,1^{\mathrm{ab}}$ \\
\hline 0,001 & $52,5 \pm 7,4^{\mathrm{bc}}$ & $50,0 \pm 8,3^{\mathrm{b}}$ & $37,5 \pm 9,7^{\mathrm{ab}}$ & $32,5 \pm 9,6^{\mathrm{ab}}$ & $41,2 \pm 8,9^{\mathrm{ab}}$ & $47,5 \pm 9,7^{\mathrm{ab}}$ & $50,0 \pm 7,7^{\mathrm{b}}$ & $62,5 \pm 20,6$ & $15,1^{\mathrm{ab}}$ \\
\hline 0,0001 & $38,7 \pm 7,4^{\mathrm{ab}}$ & $45,0 \pm 8,3^{\mathrm{ab}}$ & $40,0 \pm 9,7^{\mathrm{ab}}$ & $37,5 \pm 9,6^{\mathrm{ab}}$ & $42,5 \pm 8,9^{\mathrm{ab}}$ & $61,2 \pm 9,7^{b}$ & $72,5 \pm 7,7^{b}$ & $76,2 \pm 9,5$ & $20,4^{\mathrm{b}}$ \\
\hline 0,00001 & $40,0 \pm 7,4^{\mathrm{ab}}$ & $32,5 \pm 8,3^{\mathrm{ab}}$ & $26,2 \pm 9,7^{\mathrm{ab}}$ & $21.2 \pm 9,6^{\mathrm{ab}}$ & $26,2 \pm 8,9^{a b}$ & $37,5 \pm 9,7^{\mathrm{ab}}$ & $53,7 \pm 7,7^{\mathrm{b}}$ & $85,0 \pm 10,8$ & $25,0^{\mathrm{b}}$ \\
\hline 0,000001 & $50,0 \pm 7,4^{\mathrm{bc}}$ & $48,7 \pm 8,3^{b}$ & $45,0 \pm 9,7^{\mathrm{ab}}$ & $42,5 \pm 9,6^{\mathrm{ab}}$ & $43,7 \pm 8,9^{\mathrm{ab}}$ & $55,0 \pm 9,7^{b}$ & $63,7 \pm 7,7^{\mathrm{b}}$ & $76,2 \pm 13,8$ & $21,5^{b}$ \\
\hline 0,0 (agua) & $11,2 \pm 7,4^{\mathrm{a}}$ & $8,7 \pm 8,3^{\mathrm{a}}$ & $7,5 \pm 9,7^{\mathrm{a}}$ & $6,2 \pm 9,6^{\mathrm{a}}$ & $6,2 \pm 8,9^{a}$ & $6,2 \pm 9,7^{\mathrm{a}}$ & $8,7 \pm 7,7^{\mathrm{a}}$ & $10,0 \pm 4,1$ & $2,5^{\mathrm{a}}$ \\
\hline
\end{tabular}

$* \mu=$ Media. D. E. = Desviación estándar. E. E.= Error experimental. Letras iguales en la misma columna no difieren estadísticamente.

de $1,0 \%$ de eugenol de las 3 a $12 \mathrm{~h}$ presenta mayor porcentaje de repelencia de 56,2 - 77,5\%, y de las 24 a 72 h la concentración de $0,000001 \%$ causa mayor repelencia, que alcanza hasta 97,5 \%. Estos resultados son interesantes desde el punto de vista de la concentración a utilizar, ya que a menor concentración habrá menor presión de selección en la mosca blanca, mínimo impacto en el ambiente, más economía y nulos efectos en la salud humana. Además, la repelencia de $T$. vaporariorum por el eugenol en todas las concentraciones se mantiene a través del tiempo. A las 3 y $72 \mathrm{~h}$ la repelencia promedio de las siete concentraciones es de 50,2 y $60,7 \%$ respectivamente, con una disminución de $16,4 \%$ de la repelencia a las $6 \mathrm{~h}$, y se incrementa $22,8 \%$ a las $72 \mathrm{~h}$. El efecto del eugenol a bajas concentraciones, es un indicador que los órganos sensoriales de la mosca blanca no se saturan y por esta razón tienen mayor efecto de repelencia, afectando el sistema nervioso central octopaminérgico (Miyazawa et al. 1997; Enan 2005). Se infiere que $0,000001 \%$ de eugenol es más efectiva por repeler de 38,7 a 97,5\% de adultos de mosca blanca $T$. vaporariorum.

La repelencia de $T$. vaporariorum con metil eugenol comercial (Sigma-Aldrich) a las concentraciones de 1,0 a
$0,000001 \%$ va de 21,2 a $85,0 \%$ (Tabla 12 ). Con las concentraciones de 0,0001 a $0,000001 \%$, que son las más bajas, la repelencia es menor de $50 \%$ en las primeras $12 \mathrm{~h}$, pero a partir de las $24 \mathrm{~h}$ ésta se incrementó hasta alcanzar 85,0\% de repelencia a las $72 \mathrm{~h}$. Esto indica que las bajas concentraciones de metil eugenol tienen mejor efecto repelente de la mosca blanca, posiblemente porque no saturan sus órganos quimiorreceptores y hay persistencia sutil en el ambiente, afectando los receptores de la octopamina del sistema nervioso central (Miyazawa et al. 1997; Enan 2005). Se infiere que la concentración de metil eugenol $0,000001 \%$ es mejor por repeler de 42,5 a $76,2 \%$ de adultos de mosca blanca.

El compuesto $\beta$-cariofileno comercial (Sigma-Aldrich) a la concentración de 1,0\% causa toxicidad a las hojas de tomate desde el inicio del experimento; sin embargo, en la Tabla 13 se observa que las concentraciones de 0,1 a $0,000001 \%$ causan de 21,2 a $100 \%$ de repelencia de adultos de mosca blanca T. vaporariorum de las 3 a $72 \mathrm{~h}$. Esto indica que el compuesto $\beta$-cariofileno se debe utilizar a concentraciones por debajo de $1,0 \%$ para evitar la toxicidad de las plantas y lograr la repelencia de los adultos de mosca blanca, el cual se mantienen a

Tabla 13. Repelencia (\%) de adultos de mosca blanca Trialeurodes vaporariorum mediante $\beta$-cariofileno a concentraciones de 1,0 a $0,000001 \%$.

\begin{tabular}{|c|c|c|c|c|c|c|c|c|c|c|c|c|c|c|}
\hline Tiempo & 3 & & 4 & & 5 & & 6 & & 12 & 24 & & 48 & & $72 \mathrm{~h}$ \\
\hline Conc. (\%) & ${ }^{*} \boldsymbol{\mu}+$ D. E. & rango & $\boldsymbol{\mu}+$ D. E. & rango & $\boldsymbol{\mu}+$ D. E. & rango & $\boldsymbol{\mu}+$ D. E. & rango & $\boldsymbol{\mu}+\mathbf{E} . \mathbf{E}$ & $\boldsymbol{\mu}+$ D. E. & rango & $\boldsymbol{\mu}+$ D. E. & rango & $\boldsymbol{\mu}+$ E. E. \\
\hline 1,0 & - & & - & & - & & - & & - & - & & - & & - \\
\hline 0,1 & $50,0 \pm 17,8$ & $17,4^{\mathrm{b}}$ & $100 \pm 0,0$ & $18,0^{\mathrm{bc}}$ & $40,0 \pm 9,1$ & $19,1^{\mathrm{bc}}$ & $46,2 \pm 13,1$ & $21,1^{\mathrm{bc}}$ & $46,2 \pm 6,8^{b}$ & $48,7 \pm 18,0$ & $17,6^{\mathrm{bc}}$ & $50,0 \pm 24,8$ & $14,9^{\mathrm{ab}}$ & $78,7 \pm 4,5^{b}$ \\
\hline 0,01 & $53,7 \pm 13,1$ & $19,4^{\mathrm{bc}}$ & $40,0 \pm 7,1$ & $18,0^{\mathrm{bc}}$ & $35,0 \pm 7,1$ & $16,1^{\mathrm{ab}}$ & $36,2 \pm 6,3$ & $17,2^{\mathrm{b}}$ & $40,0 \pm 6,8^{b}$ & $45,0 \pm 24,1$ & $13,6^{\mathrm{ab}}$ & $52,5 \pm 20,2$ & $12,7^{\mathrm{ab}}$ & $93,7 \pm 4,5^{b}$ \\
\hline 0,001 & $62,5 \pm 19,4$ & $21,7^{\mathrm{bc}}$ & $55,0 \pm 23,8$ & $21,1^{\mathrm{bc}}$ & $51,2 \pm 23,9$ & $20,7^{\mathrm{bc}}$ & $51,2 \pm 25,3$ & $21,1^{\mathrm{bc}}$ & $53,7 \pm 6,8^{b}$ & $53,7 \pm 24,6$ & $18,5^{\mathrm{bc}}$ & $61,2 \pm 18,9$ & $16,9^{\mathrm{b}}$ & $76,2 \pm 4,5^{b}$ \\
\hline 0,0001 & $43,7 \pm 32,8$ & $14,5^{\mathrm{ab}}$ & $33,7 \pm 25,9$ & $13,6^{\mathrm{ab}}$ & $26,2 \pm 32,0$ & $10,9^{\mathrm{ab}}$ & $26,2 \pm 28,7$ & $11,4^{\mathrm{ab}}$ & $36,2 \pm 6,8^{a b}$ & $53,7 \pm 20,6$ & $17,9^{\mathrm{bc}}$ & $65,0 \pm 10,0$ & $18,2^{\mathrm{bc}}$ & $93,7 \pm 4,5^{b}$ \\
\hline 0,00001 & $48,7 \pm 9,5$ & $16,7^{\mathrm{b}}$ & $42,5 \pm 9,6$ & $19,0^{\mathrm{bc}}$ & $40,0 \pm 7,1$ & $19,5^{\mathrm{bc}}$ & $36,2 \pm 6,3$ & $17,2^{\mathrm{b}}$ & $40,0 \pm 6,8^{b}$ & $46,2 \pm 11,1$ & $16,0^{\mathrm{b}}$ & $62,5 \pm 5,0$ & $17,7^{\mathrm{bc}}$ & $93,7 \pm 4,5^{b}$ \\
\hline 0,000001 & $28,7 \pm 7,5$ & $9,1^{\text {ab }}$ & $22,5 \pm 10,4$ & $9,0^{\mathrm{ab}}$ & $23,7 \pm 11,1$ & $10,5^{\mathrm{ab}}$ & $21,2 \pm 10,3$ & $9,4^{\mathrm{ab}}$ & $35,0 \pm 6,8^{\mathrm{ab}}$ & $43,7 \pm 4,8$ & $15,4^{\mathrm{ab}}$ & $62,5 \pm 5,0$ & $18,5^{\mathrm{bc}}$ & $83,7 \pm 4,5^{b}$ \\
\hline 0,0 (agua) & $10,0 \pm 4,1$ & $2,6^{\mathrm{a}}$ & $7,5 \pm 2,9$ & $2,7^{\mathrm{a}}$ & $8,7 \pm 4,8$ & $4,6^{\mathrm{a}}$ & $7,5 \pm 2,9$ & $4,0^{\mathrm{a}}$ & $6,2 \pm 6,8^{a}$ & $7,5 \pm 2,9$ & $2,5^{\mathrm{a}}$ & $5,0 \pm 0,0$ & $2,5^{\mathrm{a}}$ & $8,7 \pm 4,5^{\mathrm{a}}$ \\
\hline
\end{tabular}

${ }^{*} \mu=$ Media. D. E. = Desviación estándar. E. E.= Error experimental. Letras iguales en la misma columna no difieren estadísticamente. No se cuantificó repelencia de adultos de mosca blanca debido a la fitotoxicidad en tomate desde las $3 \mathrm{~h}$. 
través del tiempo, ya que a las $72 \mathrm{~h}$ se incrementa la repelencia en $38,7 \%$. Aunque se logra repelencia total al $0,1 \%$ a las $4 \mathrm{~h}$, la concentración $0,000001 \%$ es más efectiva por repeler de 21,2 a $83,7 \%$ de adultos de mosca blanca.

El eugenol y metil eugenol a concentraciones de 0,000001 a $1,0 \%$ y $\beta$-cariofileno de 0,00001 a $0,1 \%$, causan de 18,7 a 100 $\%$ de repelencia en adultos de mosca blanca $T$. vaporariorum. La concentración de $0,000001 \%$ de eugenol y metil eugenol, se consideran mas efectivas por repeler 63,1 y $63,1 \%$ en promedio de adultos de mosca blanca. Sin embargo, aunque el compuesto $\beta$-cariofileno con esta concentración repele más de $40,1 \%$ de los adultos, se debe tener cuidado al utilizar concentraciones por encima del $1,0 \%$, porque causan toxicidad a las hojas de tomate. Por otro lado, no se tienen reportes de la aplicación de estos compuestos en el manejo de T. vaporariorum, pero se han aplicado para el manejo de otras especies de insectos causando repelencia y mortalidad. Se usó contra la hormiga roja Solenopsis invicta, Buren, 1972 (Hymenoptera: Formicidae), a concentraciones de 3 a $12 \mathrm{mg} . \mathrm{cm}^{-2}$ causando 99 y $100 \%$ de repelencia y mortalidad a las 3 y 6 h, respectivamente (Kafle y Jen 2013), contra la chicharra Cacopsylla chinensis Yang y Li, 1981 (Hemiptera: Psyllidae) con $\mathrm{DL}_{50}$ de $0,673 \mu$ g. adulto $^{-1}$ y $1,668 \mu$ g.ninfa ${ }^{-1}$. Además, con la concentración de $2,4 \%$ se reduce $66,18 \%$ la población (Bao-Liang et al. 2015). Para el mosquito A. aegypti la $\mathrm{CL}_{50}$ es de $9,086 \%$ de eugenol (Schalcher et al. 2014) y para Reticulitermes chinensis Snyder, 1949 (Isoptera: Rhinotermitidae) es de 1,21 \% (Xie et al. 2015), en $R$. speratus, otra especie de termitas, las concentraciones de 0,06 a 2,0 \% de eugenol y metil eugenol causaron $100 \%$ de mortalidad (Seon-Mi et al. 2009). El eugenol a la concentración de 3,0 y 1,3\% causó toxicidad de contacto y redujo el consumo de alimento en el gorgojo del maíz S. zeamais; a 3,5 y $9,9 \%$ disminuye el consumo de alimento en larvas y adultos de $T$. castaneum (Huang et al. 2002). El metil eugenol, actúa como inhibidor de la alimentación y es repelente de insectos (Tan y Nishida 2012). Las concentraciones de 0,$0005 ; 1,0 ; 1,5$ y $2,0 \%$ de $\beta$-cariofileno, causan $100 \%$ de mortalidad en termitas obreras (Il-Kwon y Sang-Chul 2005; Seon-Mi et al. 2009); sin embargo, Zoubiri y Baaliouamer (2014), mencionan que es necesario estudiar los efectos de los extractos y aceites esenciales como un todo, en lugar de sus principales componentes. Rodríguez (2004) indica que en el conjunto de compuestos del extracto o aceite esencial se encuentran potenciadores o que actúan como sinergistas.

Efectividad del clavo y pimienta. En la comparación de la repelencia de los extractos crudos, aceites esenciales y compuestos secundarios de clavo y pimienta no se observa efecto claro de concentración-efectividad, lo que está acorde con Pickett et al. (1997), quienes mencionan que las substancias vegetales no muestran este efecto; la respuesta de la mosca blanca no aumenta al incrementar la concentración del estímulo; esto se observa en las evaluaciones de clavo y pimienta. Se esperaba que la mayor repelencia de adultos de mosca blanca, se provocara con los compuestos secundarios, lo cual no sucedió; en contraste; estos compuestos causaron menor repelencia que los extractos crudos y aceites esenciales, logran repeler 51,0\% de los adultos. De los aceites esenciales, se esperaba mayor repelencia que los extractos crudos, resultan similares en efectividad. Los extractos crudos en diclorometano y hexano, aceites esenciales de clavo y pimienta, es posible que tengan los mismos compuestos secundarios, ya que causaron repelencia similar; desde el punto de vista de acción en el ambiente, los extractos en diclorometano y hexano no son recomendables, por su estabilidad y persistencia, debido a su polaridad, y someterán mayor presión de selección a la mosca blanca, la cual tiene la facultad de generar resistencia rápidamente. Los aceites esenciales, por su naturaleza aromática se volatilizan rápidamente y altas concentraciones causan fitotoxicidad. Además, están poco disponibles en el mercado, su extracción es más difícil y los costos son altos, porque requieren de técnicas y equipos sofisticados, que no están al alcance de los productores.

Los extractos crudos en metanol y etanol de clavo y pimienta, mostraron repelencia similar; pero debido a la toxicidad oral que produce el metanol en el ser humano no se recomienda como disolvente (Rivera y Lima 2013); además tiene poca disponibilidad en el mercado, y requiere de instalaciones con especificaciones especiales para su almacenamiento (Methanol Institute 2013). Se recomienda el uso de etanol como disolvente, con mayor disponibilidad en el mercado, amigable con el ambiente y se permite en las normas de la agricultura orgánica (Diario Oficial de la Federación 1997). Con los resultados de repelencia ocasionados por los extractos de clavo y pimienta, las sustancias extraídas con etanol no se evaporan con la temperatura, y además de mantener el efecto lo incrementan a las $72 \mathrm{~h}$, observando mayor efectividad con el extracto de clavo. Al considerar el porcentaje de repelencia provocado por las concentraciones de los extractos, se observa que no hay correlación logarítmica entre concentración-efectividad, por tanto, es importante iniciar el manejo de las poblaciones de mosca blanca con la concentración baja $(0,000001 \%)$, para disminuir riesgos ecológicos, costos de producción, contaminación y acumulación de residuos en los productos cosechados.

\section{Conclusiones}

La aplicación de extractos crudos, aceites esenciales y compuestos puros de referencia de clavo y pimienta a concentraciones de 1,0 a $0,000001 \%$, causan en promedio por tiempo de muestreo de 32,5 a 90,2 \% de repelencia de adultos de mosca blanca $T$. vaporariorum de las 3 a las $72 \mathrm{~h}$. Se considera más eficiente en la repelencia de la mosca blanca la concentración de 0,000001 , ya que logran repeler de 21,2 a $97,5 \%$, y somenten a menor presión de selección a la mosca blanca, además su efecto se mantiene a través de los tiempos de muestreo hasta las $72 \mathrm{~h}$. De acuerdo al tipo de extracción se recomienda utilizar los extractos crudos que alcanzan repelencias de 38,7 a $96,2 \%$ de las 3 a las $72 \mathrm{~h}$, especialmente de extracto crudo de ambas plantas extraidos con etanol con repelencia de 38,7 a $87,5 \%$, ya que dicho solvente es mas fácil de conseguir y está considerado como solvente en las normas de la agricultura orgánica.

\section{Literatura citada}

ABO-EL-SAAD, M. M.; AL AJLAN, A. M.; AL-EID, M. A.; BOUKHOWH, I. A. 2011. Repellent and fumigant effects of essential oil from clove buds Syzygium aromaticum L. against Tribolium castaneum (Herbst) (Coleoptera: Tenebrionidae). Journal of Agricultural Science and Technology A 1: 613-620. DOI: 10.17265/2161-6256/2011.08A.019 http://www.davidpublisher. org/Public/uploads/Contribute/55c9adc900ec5.pdf

BAGAVAN, A.; RAHUMAN A. A. 2011. Evaluation of larvicidal activity of medicinal plant extracts against three mosquito vectors. Asian Pacific Journal of Tropical Medicine 4 (1): 29-34. https://doi.org/10.1016/S1995-7645(11)60027-8 
BALDIN, E. L. L.; AGUIAR, G. P.; FANELA, T. L. M.; SOARES, M. C. E.; GROPPO, M.; CROTTI, A. E. M. 2015. Bioactivity of Pelargonium graveolens essential oil and related monoterpenoids against sweet potato whitefly, Bemisia tabaci biotype B. Journal of Pesticide Pest Science 88 (1): 191-199. https://doi. org/10.1007/s10340-014-0580-8

BALDIN, E. L. L.; CROTI, A. E. M.; WAKABAYASHI, K. A. L.; SILVA, J. P. G. F.; AGUIAR, G. P.; SOUZA, E. S.; VENEZIANI, R. C. S.; GROPPO, M. 2013. Plant-derived essential oils affecting settlement and oviposition of Bemisia tabaci (Genn.) biotype B on tomato. Journal of Pesticide Pest Science 86 (2): 301-308. https://doi.org/10.1007/s10340-012-0462-X

BAO-LIANG, T.; QI-ZHI, L.; ZHI-LONG, L.; PENG, L.; JIEWEN, W. 2015. Insecticidal potential of clove essential oil and its constituents on Cacopsylla chinensis (Hemiptera: Psyllidae) in laboratory and field. Journal of Economic Entomology 108 (3): 957-961. https://doi.org/10.1093/jee/tov075

BIRGÜCÜ, A. K.; ÇELIKPENÇE, Y.; AKDAŞ, A.; GÖKKAYA, S.; KARACA, I. 2016. Effects of different essential oils on oviposition behavior of Trialeurodes vaporariorum. Turkish Bulletin of Entomology 6 (3): 213-220. https://doi.org/10.16969/teb.31464

CAMARILLO, R. G.; ORTEGA, A. L. D.; SERRATO, C. M. A.; RODRÍGUEZ, H. C. 2009. Actividad biológica de Tagetes filifolia (Asteraceae) en Trialeurodes vaporariorum, (Hemiptera: Aleyrodidae). Revista Colombiana de Entomología 35 (2): 177184. Disponible en: http://www.scielo.org.co/pdf/rcen/v35n2/ v35n2a12.pdf

CARAPIA, R. V. E.; CASTILLO-GUTIÉRREZ, A. 2013. Estudio comparativo sobre la morfología de Trialeurodes vaporariorum (Westwood) y Bemisia tabaci (Gennadius) (Hemiptera: Aleyrodidae). Acta Zoologica Mexicana (n.s.) 29 (1): 178-193. https:// doi.org/10.21829/azm.2013.291394

CARDONA, C.; RODRÍGUEZ, I. V.; BUENO, J. M.; TAPIA, X. 2005. Biología y manejo de la mosca blanca Trialeurodes vaporariorum en habichuela y fríjol. Centro Internacional de Agricultura Tropical (CIAT); Departament for International Development (DFID). 50 p. Disponible en: http://ciat-library.ciat. cgiar.org/Articulos_Ciat/Car\%C3\%A1tula.pdf [Fecha revisión: 18 junio 2017].

CHAO, L. X.; FENG, H. J.; ZHOU, L.; LONG, L. Z. 2014. Evaluation of fumigant toxicity of essential oils of Chinese medicinal herbs against Bemisia tabaci (Gennadius) (Hemiptera: Aleyrodidae). Journal of Entomology and Zoology Studies 2 (3): 164-169. https://www.entomoljournal.com/archives/2014/ vol2issue3/PartC/50-168.pdf

CRUZ-ESTRADA, A. E.; RUÍZ-SÁNCHEZ, E.; GAMBOA-ANGULO, M. 2015. Activity of Eugenia winzerlingii Standl extracts on Bemisia tabaci Gennadius (Hemiptera: Aleyrodidae). Revista de Protección Vegetal 30 (1): 38. http://scielo.sld.cu/pdf/ rpv/v30s1/rpv015s15.pdf

DEHGHANI, M.; AHMADI, K. 2013. Repellence and Antioviposition activities of essential oils and aqueous extracts from five aromatic plants against greenhouse whitefly. Bulgarian Journal of Agricultural Science 19 (4): 691-696. https://www. agrojournal.org/19/04-10.pdf

DI-RIENZO， J. A.; CASANOVES，F.; BALZARINI，M. G.; GONZÁLEZ, L.; TABLADA, M.; ROBLEDO, C. W. 2013. InfoStat, versión 2013. Grupo InfoStat, FCA, Universidad Nacional de Córdoba, Argentina. Disponible en: http://www.infostat. com.ar [Fecha revisión: 15 mayo 2014].

DIARIO OFICIAL DE LA FEDERACIÓN. 1997. Norma Oficial Mexicana NOM-037-FITO-1995. Por la que se establecen las especificaciones del proceso de producción y procesamiento de productos agrícolas orgánicos. Disponible en: http://www.dof. gob.mx/nota_detalle.php?codigo $=4876443 \&$ fecha $=23 / 04 / 1997$ [Fecha revisión: 20 octubre 2017].

ENAN, E. E. 2005. Molecular and pharmacological analysis of an octopamine receptor from american cockroach and fruit fly in response to plant essential oils. Archives of Insect Biochemistry and Physiology 59 (3): 161-171. https://doi.org/10.1002/ arch. 20076

FOUAD, H. A. 2013. Bioactivity of five essential oils against Bruchidius incarnatus (Bohemann, 1833). Notulae Scientia Biologicae 5 (3): 354-359. https://doi.org/10.15835/nsb539065

GARCÍA S., M. A. 2002. Manual de Prácticas de Química Orgánica II. Primera edición. Unidad Iztapalapa, Universidad Autónoma Metropolitana. Iztapalapa, México D.F., México. 120 p.

GERLING, D. 2002. Una reinterpretación sobre las moscas blancas. Manejo Integrado de Plagas 63: 13-21. Disponible en: http:// www.sidalc.net/repdoc/A2096E/A2096E.PDF

GONZÁLEZ-COLOMA, A.; MARTIN-BENITO, D.; MOHAMED, N.; GARCÍA-VALLEJO, M. C.; SORIA, A. C. 2006. Antifeedant effects and chemical composition of essential oils from different populations of Lavandula luisieri L. Biochemical Systematics and Ecology 34 (8): 609-616. https://doi.org/10.1016/j. bse.2006.02.006

GRUNDY, D. L.; STILL, C. C. 1985. Inhibition of acetylcholinesterases by pulegone-1,2-epoxide. Pesticide Biochemistry and Physiology 23 (3): 383-388. https://doi.org/10.1016/00483575(85)90100-2

GUAN, W.; LI, S.; YAN, R.; TANG, S.; QUAN, C. 2007. Comparison of essential oils of clove buds extracted with supercritical carbon dioxide and other three traditional extraction methods. Food Chemistry 101 (4): 1558-1564. https://doi.org/10.1016/j. foodchem.2006.04.009

HO, S. H.; CHENG, L. P. L.; SIM, K.Y.; TAN, H. T. W. 1994. Potential of cloves (Syzygium aromaticum (L.) (Merr. \& Perry) as a grain protectant against Tribolium castaneum (Herbst) and Sitophilus zeamais Motsch. Postharvest Biology and Technology 4 (1): 179-183. https://doi.org/10.1016/0925-5214(94)90019-1

HUANG, Y.; SHUIT-HUNG, H.; HSIEN-CHIEH, L.; YEN-LING, Y. 2002. Insecticidal properties of eugenol, isoeguenol and methyleugenol and their effects on nutrition of Sitophilus zeamais Motsch. (Coleoptera: Curculionidae) and Tribolium castaneum (Herbst) (Coleoptera: Tenebrionidae). Journal of Stored Products Research 38 (5): 403-412. https://doi.org/10.1016/S0022474X(01)00042-X

HUSSEIN, H. S.; SALEM, M. Z. M.; SOLIMAN, A. M. 2017. Repellent, attractive, and insecticidal effects of essential oils from Schinus terebinthifolius fruits and Corymbia citriodora leaves on two whitefly species, Bemisia tabaci, and Trialeurodes ricini. Scientia Horticulturae 216: 111-119. https://doi.org/10.1016/j. scienta.2017.01.004

IL-KWON, P.; SANG-CHUL, S. 2005. Fumigant activity of plant essential oils and components from garlic (Allium sativum) and clove bud (Eugenia caryophyllata) oils against the Japanese termite (Reticulitermes speratus Kolbe). Journal of Agricultural and Food Chemistry 53 (11): 4388-4392. https://doi.org/10.1021/ jf050393r

ISIKBER, A. A.; ALMA. M. H.; KANAT, M.; KARCI, A. 2006. Fumigant toxicity of essential oils from Laurus nobilis and Rosmarinus officinalis against all life stages of Tribolium confusum. Phytoparasitica 34 (2): 167-177. https://doi. org/10.1007/BF02981317

ISLAM, M. S.; MAHBUB, H., M.; XIONG, W.; ZHANG, S. C.; LEI, C. L. 2009. Fumigant and repellent activities of essential oil from Coriandrum sativum (L.) (Apiaceae) against red flour beetle Tribolium castaneum (Herbst) (Coleoptera: Tenebrionidae). Journal of Pest Science 82 (2): 171-177. https://doi.org/10.1007/ s10340-008-0236-7

JUN-RAN, K.; PERUMALSAMY, H.; BONG-KI, S.; YOUNGJOON, A. 2012. Fumigant toxicity of plant essential oils against Camptomyia corticalis (Diptera Cecidomyiidae). Journal of Economic Entomology 105 (4): 1329-1334. https://doi.org/10.1603/ EC12049

KAFLE, L.; JEN, S. C. 2013. Toxicity and repellency of compounds from clove (Syzygium aromaticum) to red imposted imported fire ants Solenopsis invicta (Hymenoptera: Formicidae). 
Journal of Economic Entomology 106 (1): 131-135. https://doi. org/10.1603/EC12230

MENDOZA-GARCÍA， E. E.; ORTEGA-ARENAS， L. D.; PÉREZ-PACHECO， R.; RODRÍGUEZ-HERNÁNDEZ，C. 2014. Repellency, toxicity and oviposition inhibition of vegetable extracts against greenhouse whitefly Trialeurodes vaporariorum (Westwood) (Hemiptera: Aleyrodidae). Chilean Journal of Agricultural Research 74 (1): 41-48. https://doi.org/10.4067/S071858392014000100007

METHANOL INSTITUTE. 2013. Manual de manipulación segura del metanol. Disponible en: http://www.methanol.org/wpcontent/uploads/2016/06/Methanol-Safe-Handling-ManualFinal_Spanish.pdf [Fecha revisión: Octubre 2017].

MIYAZAWA, M.; WATANABE, H.; KAMEOKA, H. 1997. Inhibition of acetylcholinesterase activity by monoterpenoids with a p-menthane skeleton. Journal of Agriculture and Food Chemical Chemistry 45 (3): 677-679. https://doi.org/10.1021/jf960398b

NATHAN, S. S.; HISHAM, A.; JAYAKUMAR, G. 2008. Larvicidal and growth inhibition of the malaria vector Anopheles stephensi by triterpenes from Dysoxylum malabaricum and Dysoxylum beddomei. Fitoterapia 79 (2): 106-111. https://doi.org/10.1016/j. fitote.2007.07.013

ORTEGA, A. L. D.; SCHUSTER, D. J. 2000. Repellency to silverleaf whitefly adults. Gulf Coast Research \& Education Center. University of Florida. Bradenton, Florida, EE. UU. 2 p.

ORTIZ, C. M.; MEDINA, T. R.; VLADIVIA VALDIVIA, B. R.; ORTIZ, C. A.; ALVARADO, C. S.; RODRÍGUEZ, B. J. R. 2010. Mosquitas blancas plaga primaria de hortalizas en Nayarit. Revista Fuente 2 (5): 31-40. http://fuente.uan.edu.mx/publicaciones/02-05/4.pdf

PAVELA, R. 2009. Larvicidal property of essential oils against $C u l e x$ quinquefasciatus Say (Diptera: Culicidae). Industrial Crops and Products 30 (2): 311-315. https://doi.org/10.1016/j.indcrop.2009.06.005

PHASOMKUSOLSIL, S.; SOONWERA, M. 2011. Comparative mosquito repellency of essential oils against Aedes aegypti (Linn.), Anopheles dirus (Peyton and Harrison) and Culex quinquefasciatus (Say). Asian Pacific Journal of Tropical Biomedicine 1 (1): S113-S118. https://doi.org/10.1016/S22211691(11)60136-6

PICKETT, J. A.; WADHAMS, L. J.; WOODCOCK, C. M. 1997. Developing sustainable pest control from chemical ecology. Agriculture, Ecosystems and Environment 64 (2): 149-156. https://doi.org/10.1016/S0167-8809(97)00033-9

PITASAWAT, B.; CHAMPAKAEW, D.; CHOOCHOTE, W.; JITPAKDI, A.; CHAITHONG, U.; KANJANAPOTHI, D.; RATTANACHANPICHAI, E.; TIPPAWANGKOSOL, P.; RIYONG, D.; TUETUN, B.; CHAIYASIT, D. 2007. Aromatic plant-derived essential oil: An alternative larvicide for mosquito control. Fitoterapia 78 (3): 205-210. https://doi.org/10.1016/j. fitote.2007.01.003

RIVERA, J. L.; LIMA, R. 2013. Efecto desorbedor del metanol en la membrana celular. Revista Especializada en Ciencias Químico-Biológicas 16 (2): 93-97. https://doi.org/10.1016/S1405888X(13)72080-1

RODRÍGUEZ, H. C. 2004. Plantas atrayentes de insectos plaga. pp. 203-234. En: Tornero, C. M.; López-Olguín, J. F.; Aragón, A. G. (Eds.). Ciencias Ambientales y Agricultura. Publicación especial de la Benemérita Universidad Autónoma de Puebla. Puebla, México. Disponible en: http://www.cm.colpos.mx/cesareo/Divulgaci\%C3\%B3n/Plantas\%20atrayentes $\% 20 \mathrm{de} \% 20$ insectos\%20plaga.pdf

SALVUCCI, M. E. 2000. Sorbitol accumulation in whiteflies: Evidence for a role in protecting proteins during heat stress. Journal of Thermal Biology 25 (5): 353-361. https://doi.org/10.1016/ S0306-4565(99)00107-2

SCHALCHER, P. A. I.; SCHALCHER, P. A.G.; SOBRINHO O. P. L.; CANTANHEDE, E. K. P.; SALDANHA, S. L. F. 2014. Actividade antimicrobiana no combate as larvas do mosquito
Aedes aegypti: Homogeneização dos óleos essenciais do linalol e eugenol. Educación Química 25 (4): 446-449. https://doi. org/10.1016/S0187-893X(14)70065-5

SEON-MI, S.; KIM, J.; SANG-GIL, L.; CHANG-HOON, S.; SANG-CHUL, S.; IL-KWON, P. 2009. Fumigant antitermitic activity of plant essential oils and components from ajowan (Trachyspermum ammi) allspice (Pimenta dioica), caraway (Carum carvi), dill(Anethumgraveolens), geranium(Pelargonium graveolens) and litsea (Litsea cubeba) oils against Japanese termite (Reticulitermes speratus Kolbe). Journal of Agricultural and Food Chemistry 57 (15): 6596-6602. https://doi.org/10.1021/ jf9015416

SHARAWI, S. E.; ABD-ALLA, S. M.; OMARA, S. M.; AL-GHANDIGHAMDI, K. M. 2013. Surface contact toxicity of clove and rosemary oils against American cockroach, Periplaneta Americana (L.). African Entomology 21 (2): 324-332. https:// doi.org/10.4001/003.021.0204

SIGHAMONY, S.; ANEES, I.; CHANDRAKALA, T.; OSMANI, Z. 1986. Efficacy of certain indigenous plant products as grain protectants against Sitophilus oryzae (L.) and Rhyzopertha dominica (F.). Journal of Stored Products Research 22 (1): 2123. https://doi.org/10.1016/0022-474X(86)90042-1

TAN, K. H.; NISHIDA, R. 2012. Methyl eugenol: its occurrence, distribution, and role in nature, especially in relation to insect behavior and pollination. Journal of Insect Science 12 (56): 1-60. https://doi.org/10.1673/031.012.5601

WOLFE, G. R.; HENDRIX, D. L.; SALVUCCI, M. E. 1998. A thermoprotective role for sorbitol in the silverleaf whitefly, Bemisia argentifolii. Journal of Insect Physiology 44 (7-8): 597-603. https://doi.org/10.1016/S0022-1910(98)00035-3

WON-IL, C.; EUN-HEE, L.; BYEOUNG-RYEOL, C.; HYUNGMAN, P.; YOUNG-JOON, A. 2003. Toxicity of plant essential oils to Trialeurodes vaporariorum (Homoptera: Aleyrodidae). Journal of Economic Entomology 96 (5): 1479-1484. https://doi. org/10.1093/jee/96.5.1479

WON-SIK, C.; BYEOUNG-SOO, P.; YOUNG-HAENG, L.; DOYOUN, J. D.; HEY-YOUNG Y. H.; SUNG-EUN, L. 2006. Fumigant toxicities of essential oils and monoterpenos monoterpenes against Lycoriella mali adults. Crop Protection 25 (4): 398-401. https://doi.org/10.1016/j.cropro.2005.05.009

XIAO-WEI, W.; PING, L.; SHUESHU-SHENG, L. 2017. Whitefly interactions with plants. Current Opinion in Insect Science 19: 70-75. https://doi.org/10.1016/j.cois.2017.02.001

XIE, Y.; YANG, Z.; CAO, D.; RONG, F.; DING, H.; ZHANG, D. 2015. Antitermitic and antifungal activities of eugenol and its congeners from the flower buds of Syzygium aromaticum (clove). Industrial Crops and Products 77: 780-786. https://doi. org/10.1016/j.indcrop.2015.09.044

ZOUBIRI, S.; BAALIOUAMER, A. 2014. Potentiality of plants as source of insecticide principles. Journal of Saudi Chemical Society 18 (6): 925-938. https://doi.org/10.1016/j.jscs.2011.11.015

\section{Origen y financiación}

El presente trabajo se originó de las investigaciones realizadas para obtener el grado de Doctor en Ciencias en Fitosanidad en el área de Entomología del Colegio de Postgraduados campus Montecillo por el C. Eduardo Aguilar-Astudillo durante el periodo de 2013 a 2017, con beca PRODEP dependiente de la SEP, y el $4^{\circ}$ año con beca de manutención y estancia por CONACYT.

\section{Contribución de los autores}

El primer autor Eduardo Aguilar-Astudillo, realizó los bioensayos, toma de muestras, ordenamiento, análisis e interpretación de datos y escritura de los resultados.

Los autores Cesáreo Rodríguez-Hernández, Hiram Bravo-Mojica, Ramón Marcos Soto-Hernández, Néstor Bautista-Martínez y Francisco Guevara-Hernández, apoyaron en la interpretación de los resultados y en la revisión del escrito. 